B. OPTICAL SYSTEMS 


\title{
OPTICAL SYSTEMS FOR UV SPACE RESEARCHES
}

\author{
G. COURTÈS \\ Laboratoire d'Astronomie Spatiale du C.N.R.S., Marseille, France
}

\begin{abstract}
This paper gives a general review of the best ways to adapt the UV optical designs to the peculiar conditions of guidance of space vehicles.

(1) Conditions to obtain useful photometric direct images of the sky (stars and nebulae) with several different receptors (photographs, image tube, TV camera etc.,...). New solutions of imagery are considered.

(2) Selection of the different bandwidths of wavelengths (filters; gratings).

An example of a new optical design, the BPM filter especially adapted to the poor conditions of guidance, is described. (Wave length selection is independent of the guidance.)

The last part of this article is devoted to a review of the dispersive optical designs adapted to space research.

Conclusions are in favour of the average size space vehicles on rockets for the time being and for large telescopes in the space platforms even if their guidance is several times worse than their optical quality.
\end{abstract}

\section{Introduction}

A new field in optics has been open now for several years owing to the needs of space research. This symposium is restricted to the UV optical designs. We try to give a general idea of the particular aspects of the UV space optics. This following paper cannot be a very full report, optical instrumentation is less and less described in the scientific publications as if it were only a technical matter. In spite of that, the chance of discovery and the value of the data are deeply dependent of the quality of the optical concepts and of their good construction. It is perhaps the reason why the scientists, convinced of this obvious fact, talk so little about optical designs.

We apologize for involuntary omissions.

In space research, optical systems depend mainly on:

(1) Scientific programs;

(2) Vehicle facilities and recovery:

- weight,

- dimension,

- thermal conditions,

- mechanical constraints (vibrations).

Our first attention is to dimensional constraints which are imperative in the choice of the optical design. It is difficult, for example, to imagine a telescope having a diameter larger than the vehicle diameter.

Thus the adopted solution for the optical design will be different in the case of: planes, balloons, rockets (small and large), satellites, space stations, interplanetary platforms, and lunar stations. However, the solutions will have simplicity and ingenuity in common. They also have to be light. Dimensional constraints often force the 
adoption of additional mirrors, spoiling this simple ingenuity of the optics (Figures 10, 19). One can see that it is a very extended scientific and technical problem.

(3) Stabilization, acquisition, guidance. This third constraint is sometimes a paradox. Some modest vehicles like small rockets are equipped with high positioning and guidance, and some large satellites have no stabilization. Thus, the size of the optical system depends, for other reasons, on the vehicle. For example, the large Gemini vehicle, non-stabilized, used a small focal length and, consequently, a small diameter camera, to obtain some direct pictures and spectra from the bright stars, owing to the ability of the astronaut. We faced such a problem ourselves with the LAS* experiment S 183 in the Apollo Skylab (Fig. 6). Small rockets with stabilized platforms (ACS Space General, U.S.A.), (Sky Lark, Culham Laboratories, U.K.), (Cassiopée -

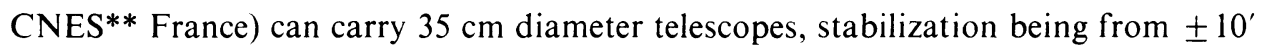
and below, 1' (Cassiopée) and a few seconds of arc with complementary fine guidance.

From an historical point of view, it is amazing to remember that the very sophisticated large astronomical satellites were conceived before any attempt was made to put a small camera on a rocket (Hallock, 1962; Davis and Rustzi, 1962).

Now, both have given results, but, after more than ten years of space research, we have only a very rough idea of how the sky looks in UV.

\section{Image of the Sky}

We will begin at the beginning and consider the very important purpose of obtaining an UV image of the sky before looking for some more difficult programs. Among the ground-based observatories, the Palomar Sky Survey and some others, such as the surveys with small cameras, have initiated many important scientific works and discoveries.

What space telescope will play a similar role?

\section{Problem of obtaining a good image of the Sky in the uV light (optical DESIGN)}

(a) Refractive elements, (quartz, magnesium fluoride, lithium fluoride, sapphire) are very few, but can be used as photoelectric tube windows, field and correcting lenses, array of lenses, etc... They can be obtained with aspherical figures (Baranne et al., 1969).

(b) Mirrors, with new good coatings (selective or of large spectral range) are available. In spite of their good reflection power, it is evidently better to use as small a number of mirrors as possible.

(c) Optical combinations. From the simple concave parabolic collector with a plate or photomultiplier in its focus (like the first Goddard rocket experiment of Kupperian et al., 1958), up to the real telescopes of two large mirrors and spectrometer like the

* LAS Laboratoire d'Astronomie Spatiale du C.N.R.S. - France.

** CNES :- Centre National d’Etudes Spatiales, France. 


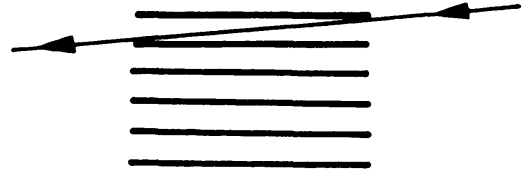

Fig. 1.

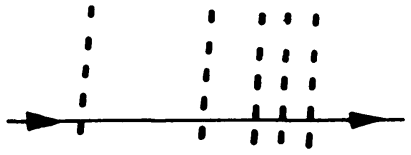

Fig. 2 .

Figs 1 and 2. Two solutions for collimators, Nb 2 prevents from stray light by reflexion effects.

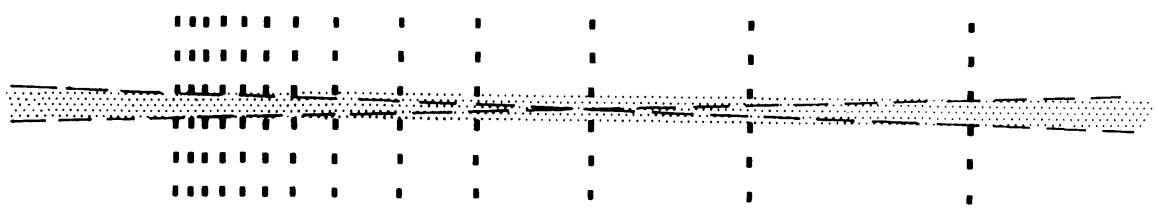

Fig. 3. Theoretical distribution of the grids is given by the expression

$$
l_{n}=\left(L-l_{n \cdot 1}\right) x+l_{n \cdot 1} .
$$

where $L$ is the total length of the instrument, and $x$ depends on the relative slit width. This example corresponds to a part of the grid repartition of the French $\mathrm{D}_{2} \mathrm{~B}$ astronomical satellite.

(To be flown by the CNES in 1973.)

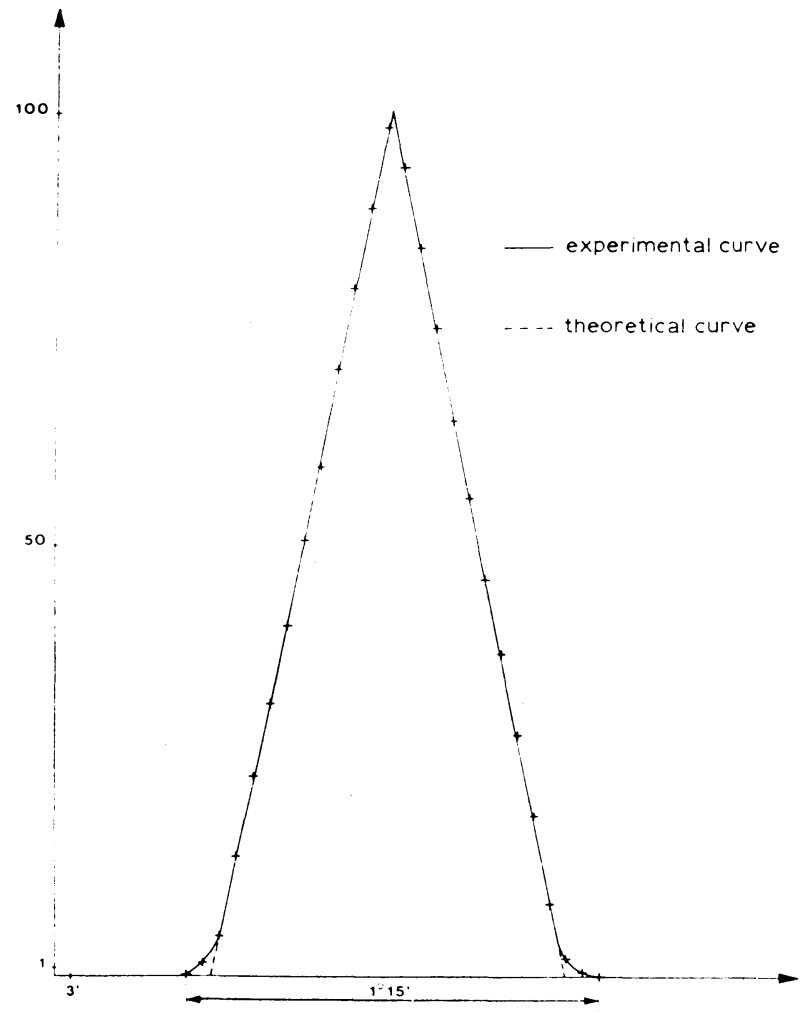

BAFFLE D.2.B

Fig. 4. Example of the baffle instrument function of $\mathrm{D}_{2} \mathrm{~B}$ satellite (Figure 3) by P. Cruvellier, M. Maucherat and M. Blanc (LAS). 
OAO telescope Hallwok, 1962; Davis and Rustzi, 1962) there are a lot of optical solutions adaptable to the astronomical problems.

\section{SELECTION OF THE DiffERENT OPTICAL SyStemS}

This selection is not limited by the fundamental optics, since telescopes with two mirrors, or one mirror and one lens (Schmidt. Maksutov, Wright) give excellent
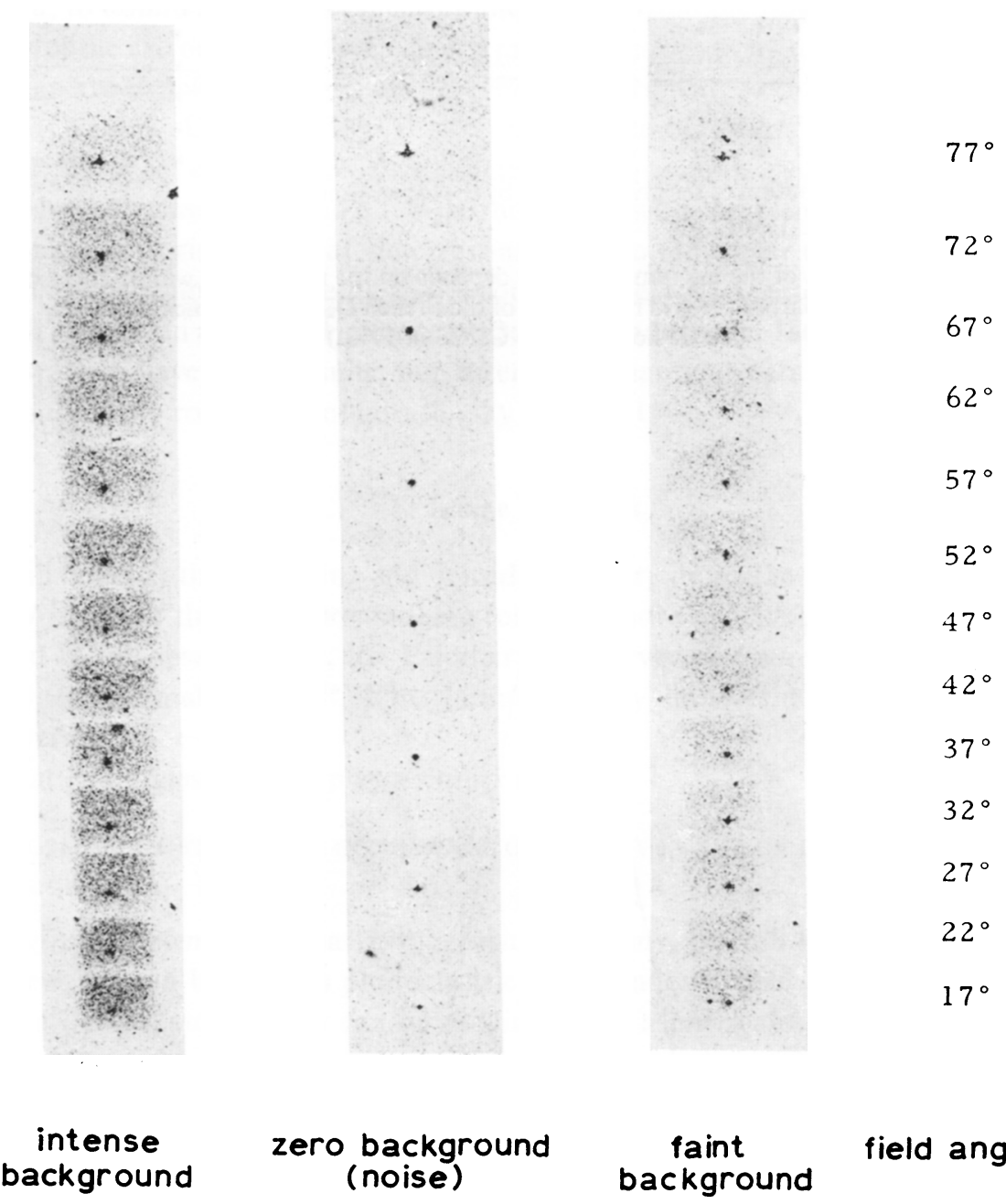

field angles

\section{PUV LAS Camera}

Fig. 5. Stellar magnitude calibration with different backgrounds and different positions in the field. total bandwidth: $2200-3200 \AA$

film: 103 a0 UV Kodak

exposure: $210 \mathrm{~s}$

enlargements:

20

(Plate from Viton and Sivan.) 


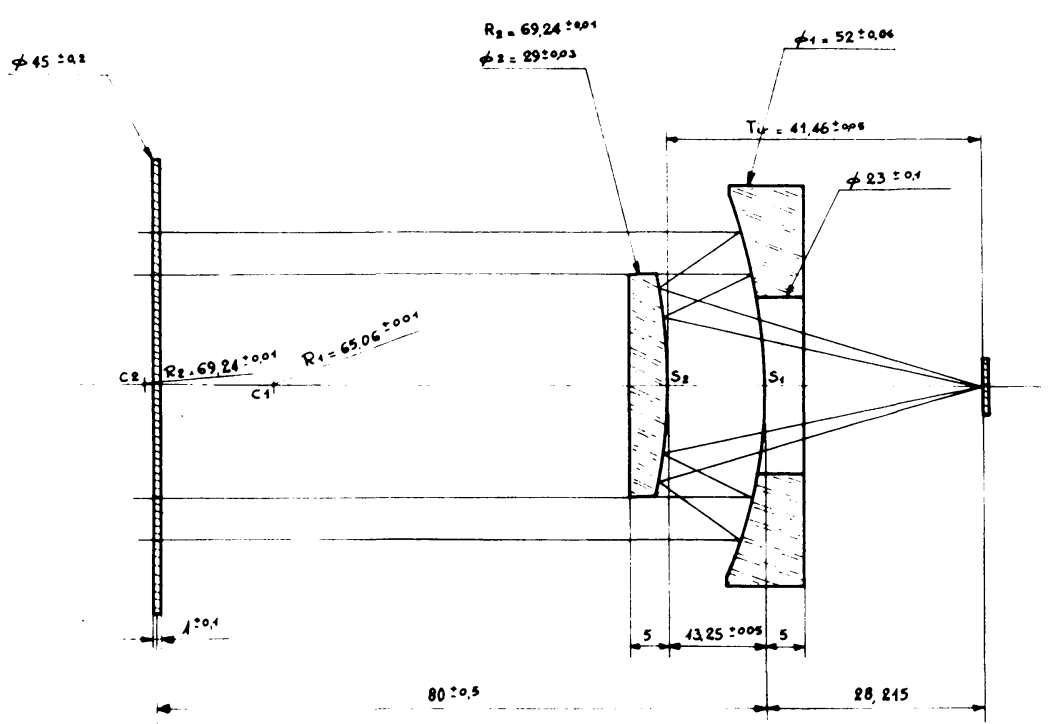

Fig. 6. $16 \mathrm{~mm}$ film recognition camera. Designed for $\mathrm{S} 183$ Apollo Skylab by M. Laget and M. Saisse.

images on a large field, but dependent on the observing conditions and constraints The most limiting constraint is stabilization; it will help us to classify the main optical systems.

\section{Generation I}

Poor acquisition needs a large field.

Poor guidance or no guidance needs improvement of the signal or sophistication of the reductions.

A solution consists of a simple collector, with photoelectric receptor and scanning (sometimes with the natural spin of the rocket) (first Goddard rocket experiment, 1957). This is a poor solution for rockets but paradoxically is still used now in some satellites, OSO (NASA), D2B (CNES), TDI(ESRO), etc....

Original peculiar design: These designs need a kind of collimator (the baffle) in order to have a convenient limitation of the field. The baffles are accurately adapted to each instrumentation. They are essentially of two kinds:

(a) honeycomb: one or two-dimensional (Figure 1) and (b) grid (Figure 2). Some examples are given by McGrath and Marwitt (1969) (Culham Laboratory and LAS), this last solution seems better to obtain the angular resolution free from reflection. The diffraction of each slit plays of course a certain part, but it is much easier to contiol than the possible reflection under grazing incidence of the honeycomb. (Figures 3 and 4 )

\section{Generation II}

Stabilized platforms of rockets (ACS Space General, Cassiopée CNES, etc....) $\mathrm{E}= \pm 15^{\prime}$ Acquisition $= \pm 1^{\circ} 5$. 
To be sure to get the star or the nebula, the optical system must give at least a field of 3 to $4^{\circ}$. The space resolution will be of $30^{\prime}$. If one wants to obtain a good photographic photometric calibration of the star image, then this image will have to be of approximately gaussian distribution (convolution of the limit cycle and the resolution of the emulsion). It is possible to have samples of photometric calibration images to compare for accurate photometry (Figure 5).

With $\mathrm{E}= \pm 15^{\prime}$, the focal length of the camera will be $f=g / 2 \mathrm{E}$, if $g \leqslant 100 \mu$ minimum spot for good photometry, $f \# 1 \mathrm{~cm}$,

In spite of so short a focal length, it is possible to obtain valuable images of the sky.

The 'Night Camera' PUV (Sivan and Viton (1970) of the LAS and the astronauts' camera in their EVA (Extra Vehicular Activities) have a very short focal length.

The TV Vidicon and super-Vidicon cameras should give similar conditions, but with an uncertain deterioration of accuracy, because of the variable number of lines, scanning a star image, and for non-uniform spatial response of the photocathode of the TV tube.

The EVA cameras have small anastigmatic lenses with an aperture of $22 \mathrm{~mm}$ and a $30^{\circ}$ field. For the Apollo Skylab, LAS designed a $16 \mathrm{~mm}$ film, star field recognition camera (two mirrors + aspheric lens) (Figure 6). (Flat field is obtained by the same radius of curvature of the two mirrors, zero Petzval sum.)

The wide field and high focal ratio camera (PUV). This solution, tried in 1967, is still

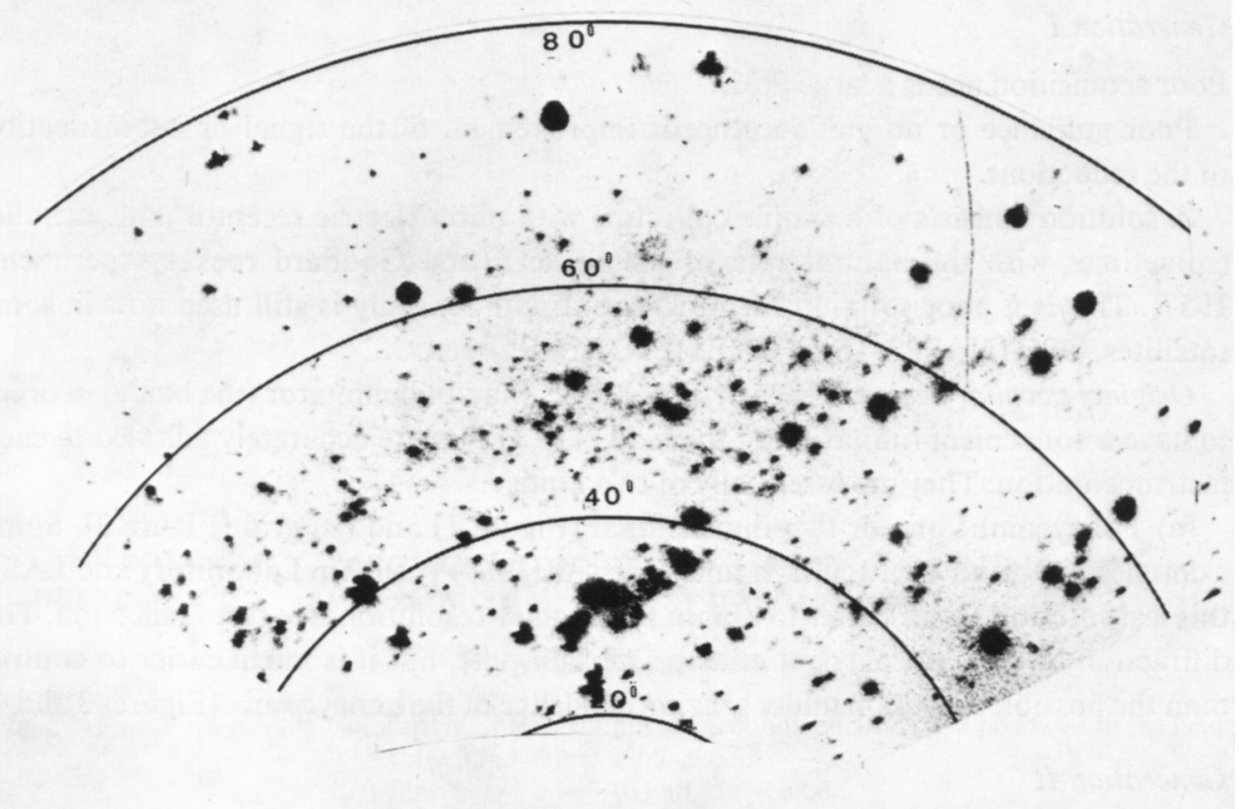

Fig. 7. $210 \mathrm{~s}$ exposure. $F / 1$ on the Milky way. One sees ORION, Pléiades and Zodiacal Light. Bandwidth 2200-3200 ̊. Plate from Viton and Sivan. 
OPTICAL SYSTEMS FOR UV SPACE RESEARCHES

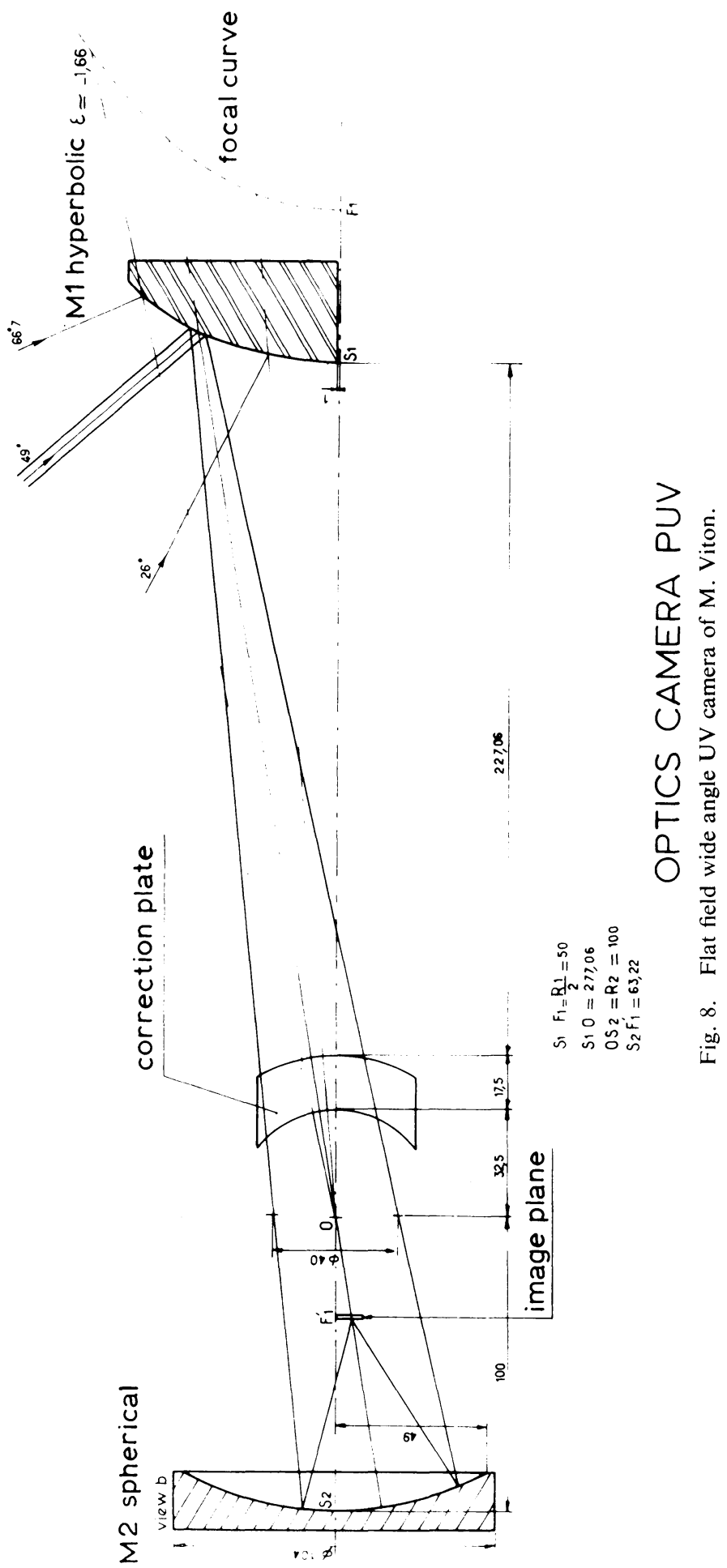


interesting since it is up to now the only one having given a wide field of the sky in UV $\left(120^{\circ} \times 80^{\circ}\right.$ for the LAS camera). (Figure 7).

LAS night camera is $F / 1, f=8 \mathrm{~mm}$. It is a Maksutov camera focused on the virtual convex image of the sky given by a hyperbolic convex mirror (Figure 8). One obtains a flat field on the film (Figure 9).

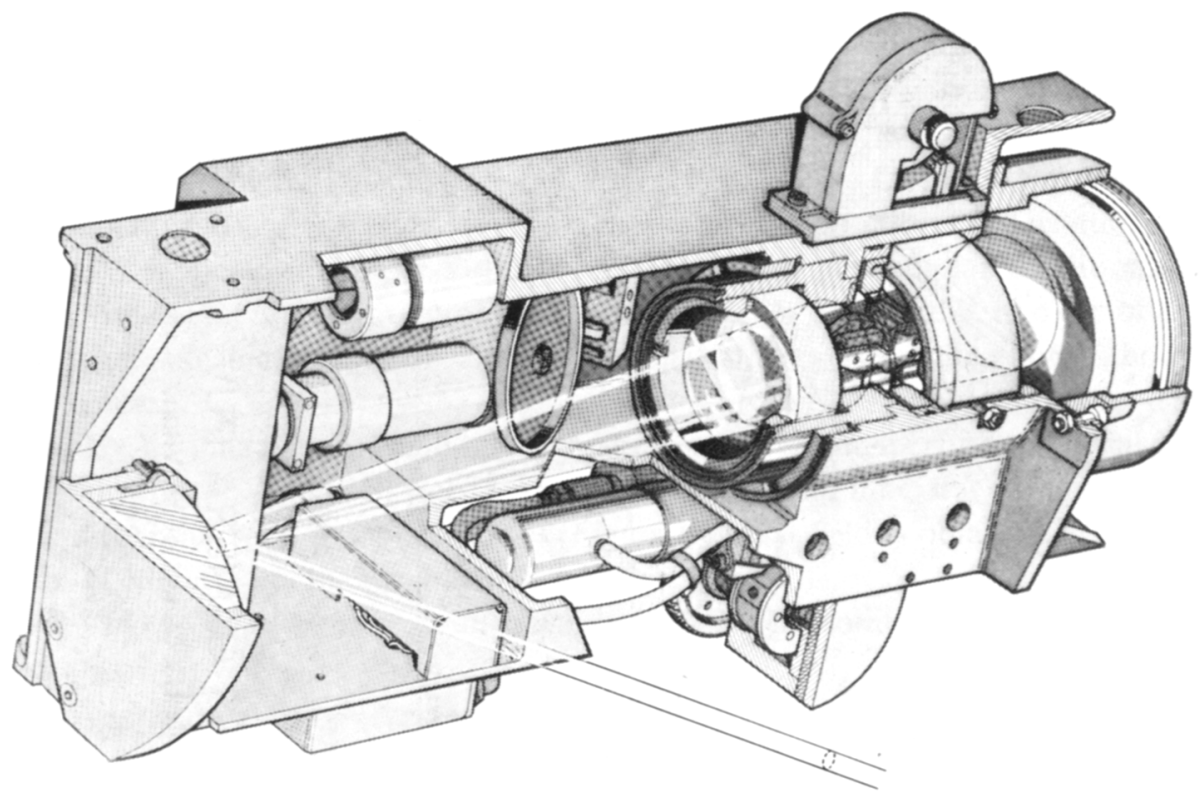

Fig. 9. The flat field wide angle camera of the LAS in its mecanical mount designed for rocket use.

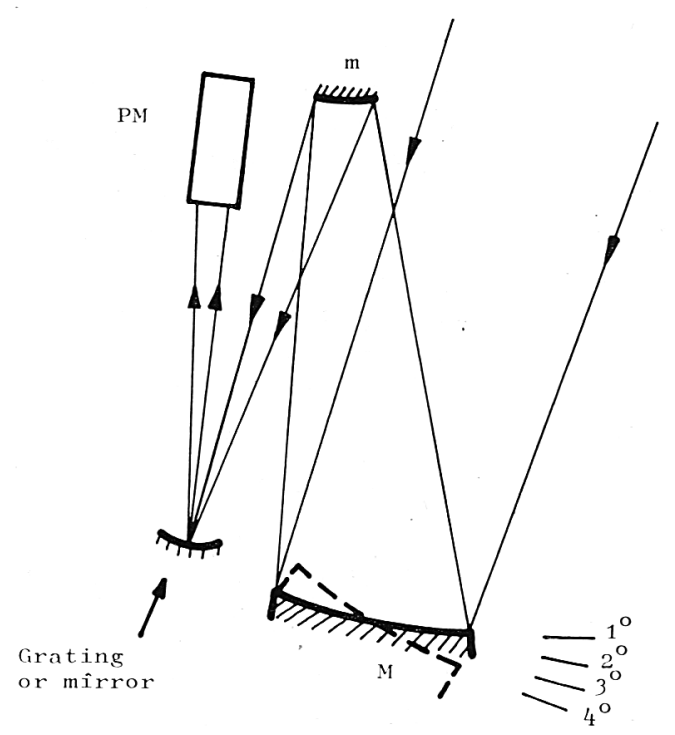

Fig. 10. LAS. Persée experiment on M31. The m mirror is needed because of the dimensional constraints. 
The magnitude $m=8$ has been reached for $O$ stars, 800 stars were detected and 250 have given colour indices with an accuracy of $\Delta m= \pm 0,2 m$ (Sivan and Viton, 1970).

Photolectric photometer. Generation II stabilization is well adapted to the photoelectric designs. The Persée (LAS) fired on the Andromeda M 31 galaxy in 1968 was of this type, with a step-by-step scanning mirror (Figure 10) visiting 16 fields of 1 square degree each since the acquisition is $\pm 1^{\circ} 5$ (Cruvellier et al., 1970) (Figure 11). This experiment gave the UV excess of M 31 confirmed by OAO Smithsonian experiment.

\section{Generation III}

Good guidance is $\pm 1^{\prime}$ and better. With the same remark as in Generation II, the focal

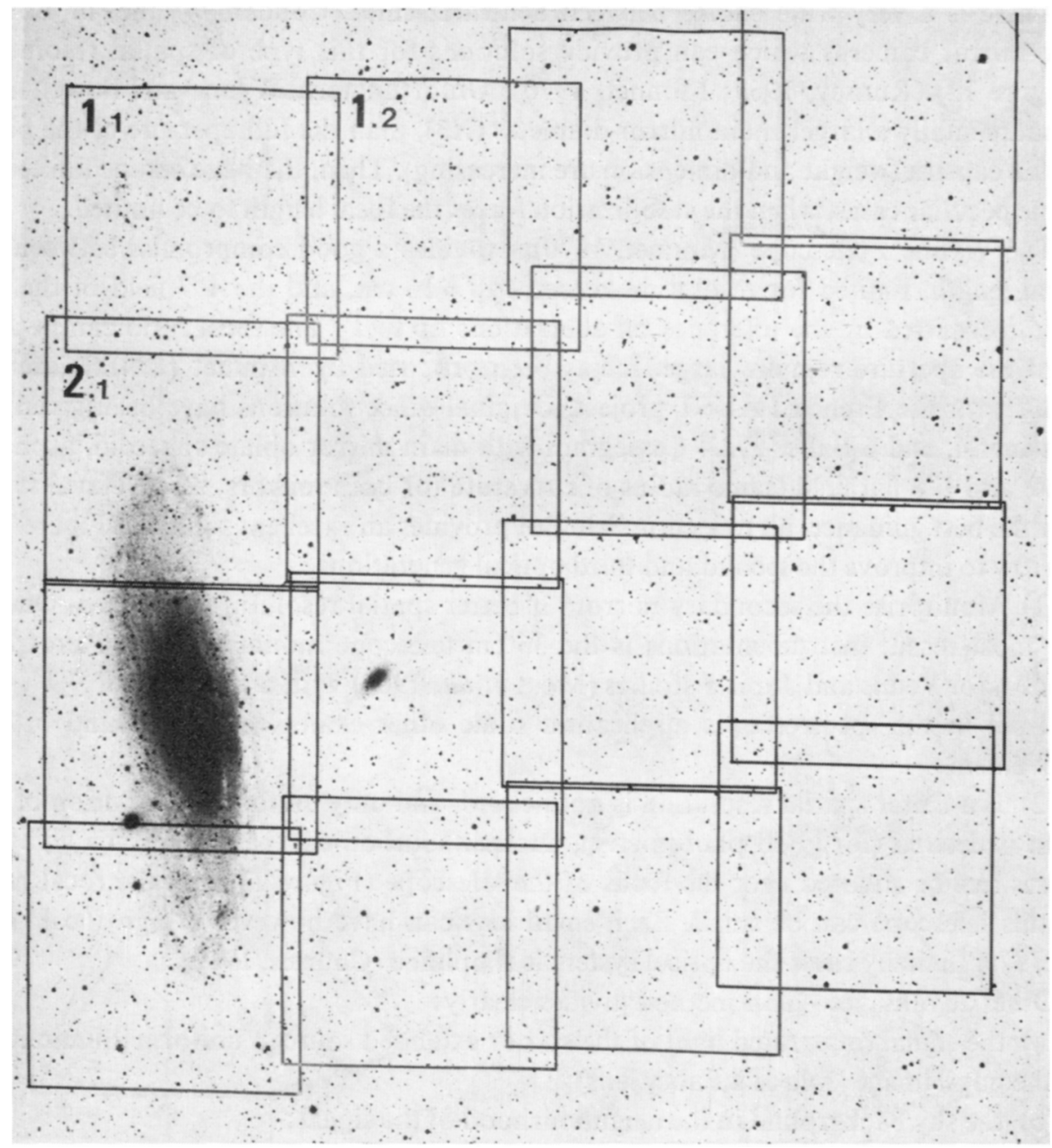

Fig. 11. Bidimensional scanning of the sky in Andromeda by the LAS. Persee Experience. See Cruvellier et al. (1970) (M 31 and NGC 205 have been observed). 
length is $f=15 \mathrm{~cm}$ and more. Good acquisition of few minutes similar to the Cassiopée stabilized platform CNES Céphée LAS experiment (Cassegrain telescope and four bandwidths monochromator is of this type).

The 1905 conventional two-mirror Schwartzschild (Burch, 1942; Runsey, 1969) telescope or the A. Couder aplanatic telescopic give a good solution for space resolution. One good example is the OAO Smithsonian telescope which has given some very well-known results (to be described here by Dr Code). The Schwartzschild telescope is an anastigmat for $d$ (distance between the mirrors) $=2 f$, with a field curvature of $\varrho=\frac{4}{7} F$ but with the flat field if $d=1.25 \mathrm{f}$. A certain amount of astigmatism appears but the compromise is good enough for this application

$\left(\begin{array}{ll}f=61 \mathrm{~cm} & \text { for OAO } \\ \varnothing=31.7 \mathrm{~cm}\end{array}\right)$.

There is a very wide choice between Schwartzschild, Couder, Wynne, or Bowen two-mirror cameras which can provide solutions for this type of spatial resolution (Figure 12) (Runsey, 1969; Monnet, 1970). Unfortunately, Wynne and Bowen have fundamentally a larger main mirror diameter (X3), than the full aperture of the pupil of the camera (weight and dimension are increasing). Then, their use can be limited to some peculiar cases, when the stabilization forces the focal length to be limited.

The Wynne's telescope (Monnet, 1970), provides a good compromise between the focal length, limited for resolution reason to $f=16 \mathrm{~cm}$, and the $4^{\circ} 7$ field of the TV tube, permitted by the tolerance on aberrations up to $1^{\prime}$. The focal ratio can be $F / 1$, then the aperture remains large. It has been proposed by Monnet (CNES internal report) for the French FAUST project. Original other solutions have been given for Mariner 4, and 6 Baker $F / 2.4$ Cassegrain with main mirror oblate spheroid, aspheric plate and $1^{\circ} 8$ flat field (same radius of curvature for both mirrors, zero Petzval sum). For the best guidance, all reflection Schmidt provides an excellent solution (Figure 12).

How to improve the second and third optical generations?

(1) Monitorise the secondary mirror* if better spatial resolution is needed. One of the most recent instrumentations is the $36 \mathrm{~cm}$ telescope mounted on an Aerospace Rocket for Venus and Jupiter studies (Moss et al., 1969) with a guidance of \pm 1 ". We will see in the spectroscopic application some other examples of this kind of improvement.

(2) If a better spatial resolution is not needed, and only uniform distribution of the light is desired (for good photometry), another solution can be chosen. An array of lenses can be situated near the focus of the telescope (Figure 13) and the focal ratio of this telescope can be small. Each small lens can have however a high focal ratio $F / 2, F / 1$. In many cases the optical system is simplified (Courtès, 1967).

Other advantages - This method divides clearly:

(a) the signal (integrated light of the star or extended source), uniform illumination of the pupil image (role of a Fabry lens);

(b) the sky background in the neighbourhood of the signal;

* Or a flat mirror such as the one of the FPL Convair 990 of NASA $33 \cdot 57$ in. E ... 10", $\left(0.5^{\prime \prime}\right.$ during a few seconds). 


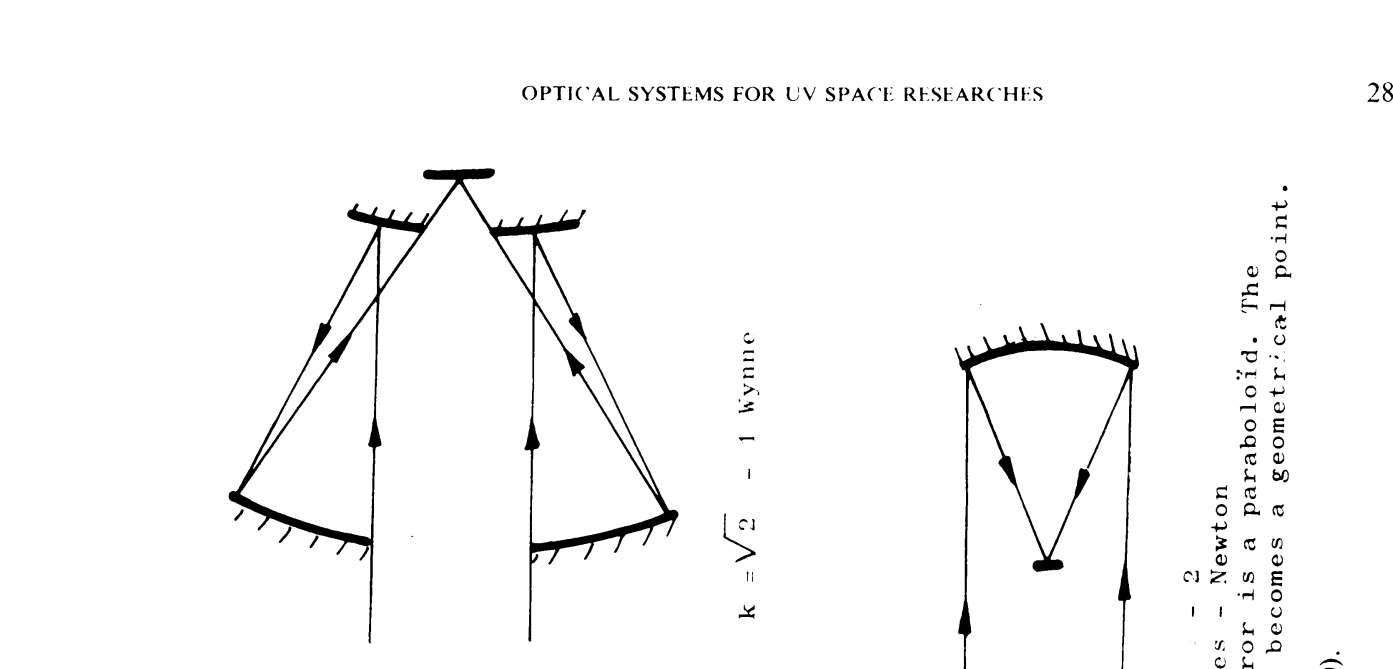

$$
\begin{aligned}
& A, B \\
& \Delta \cdot N_{0}
\end{aligned}
$$


(c) the granulation or noise of the emulsion (as well as the image tube);

(d) the point to point sensitivity control owing to a calibration of the emulsion (or of the spatially non-uniform response of the photocathode). A calibration pupil is situated by the side of the entrance pupil.

Digit image processing can be applied to take into account all these parameters giving a better photometry and a resolution defined by spatial frequency of the array of lenses (Billingsly, 1970). The image can be fully reconstructed at these resolutions as it is shown on the Moon and M 51 photographs (Figures 14 and 15). (One can judge

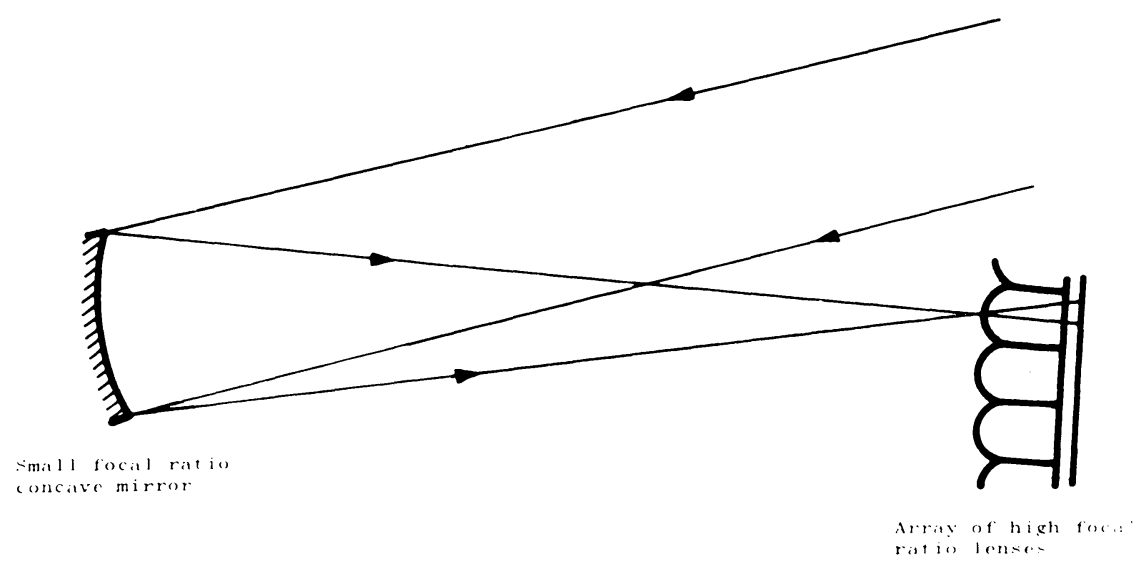

Fig. 13. Very transparent and simple solution to obtain a high focal ratio with small focal ratio collector. (Courtès, 1967.)

what very interesting information on UV light repartition in the $M 51$ spiral arms has been already given with a relatively poor guidance and optical quality $\left( \pm 6^{\prime \prime}\right)$ telescope.) The array of lenses method can be extended at the simultaneous multiband spectrophotometry (S 183 Apollo's Skylab sky panorama LAS experiment).

Generation IV (From 1" to the diffraction limit)

This generation is the absolute solution not only for technical performances but mainly to obtain the best star detection against the sky background and, something never reached on the earth, the diffraction limit for large telescopes, an evident key to new discoveries.

Recent observations of Stratoscope II (Balloon) show the diffraction ring for a 36 in. telescope on a star and on the nucleus of a Seyfert galaxy. It is the first very important step in the high spatial resolution.

Instruments of this generation are:

$\begin{array}{lll}\text { Stratoscope I } & 12 \text { in. } & 2 \mathrm{E}=0.5^{\prime \prime} \\ \text { Stratoscope II } & 36 \text { in. } & 2 \mathrm{E}=0.2^{\prime \prime}\end{array}$

Apollo telescope Mount 120 in. $2 \mathrm{E}=0.05^{\prime \prime}$ 


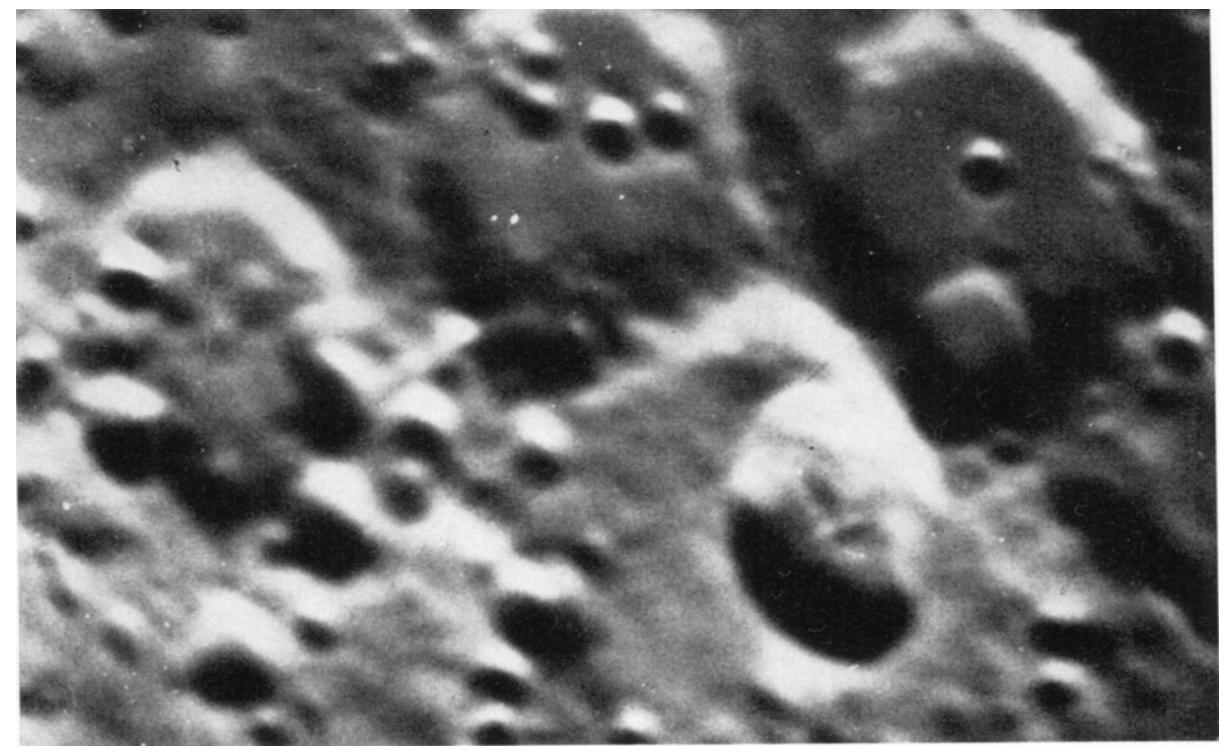

Fig. 14a. Direct picture of the Moon obtained with $F / 30$.

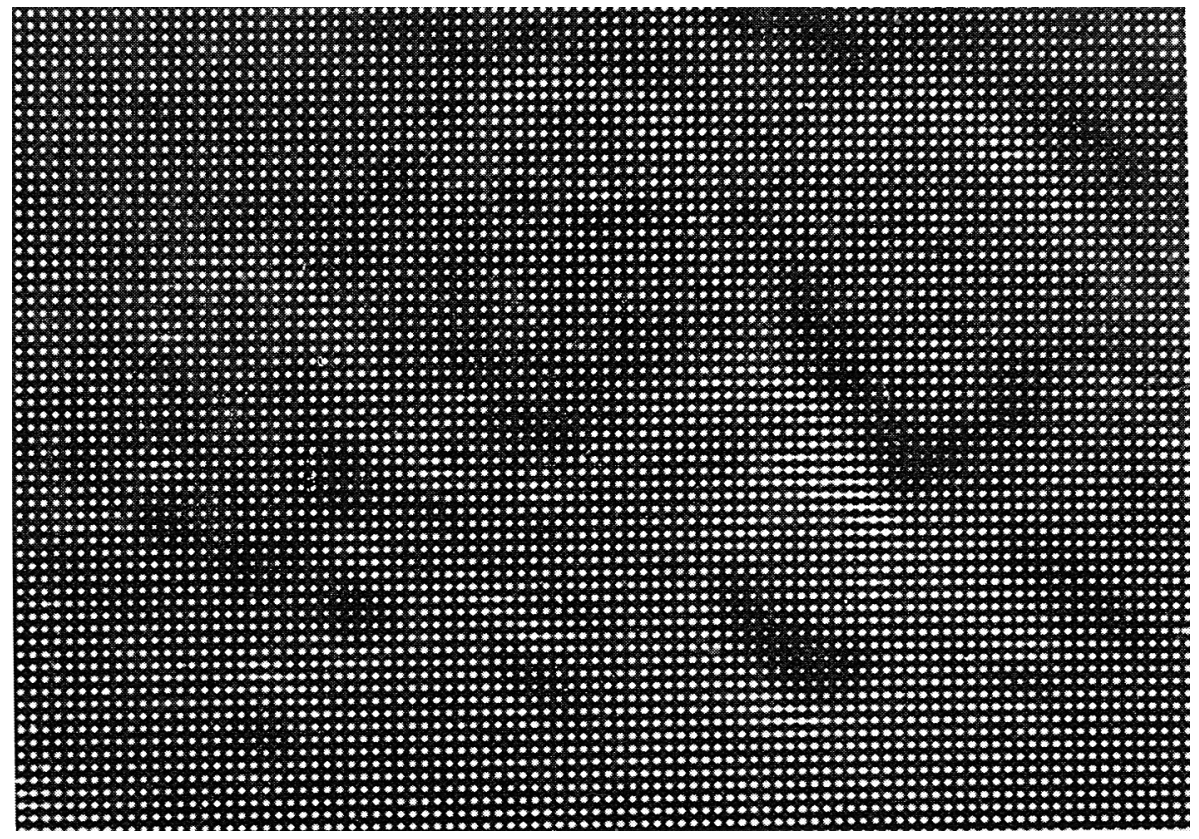

Fig. 14b. Plate obtained through a M. Bonnet array of lenses, the resolution is about 1 1"5. (a) and (b) have been obtained with the same exposure time, (b) was behind a 0.01 transmission filter. High sensitivity is obtained owing to the $F / 3$ focal ratio of the lenses. 


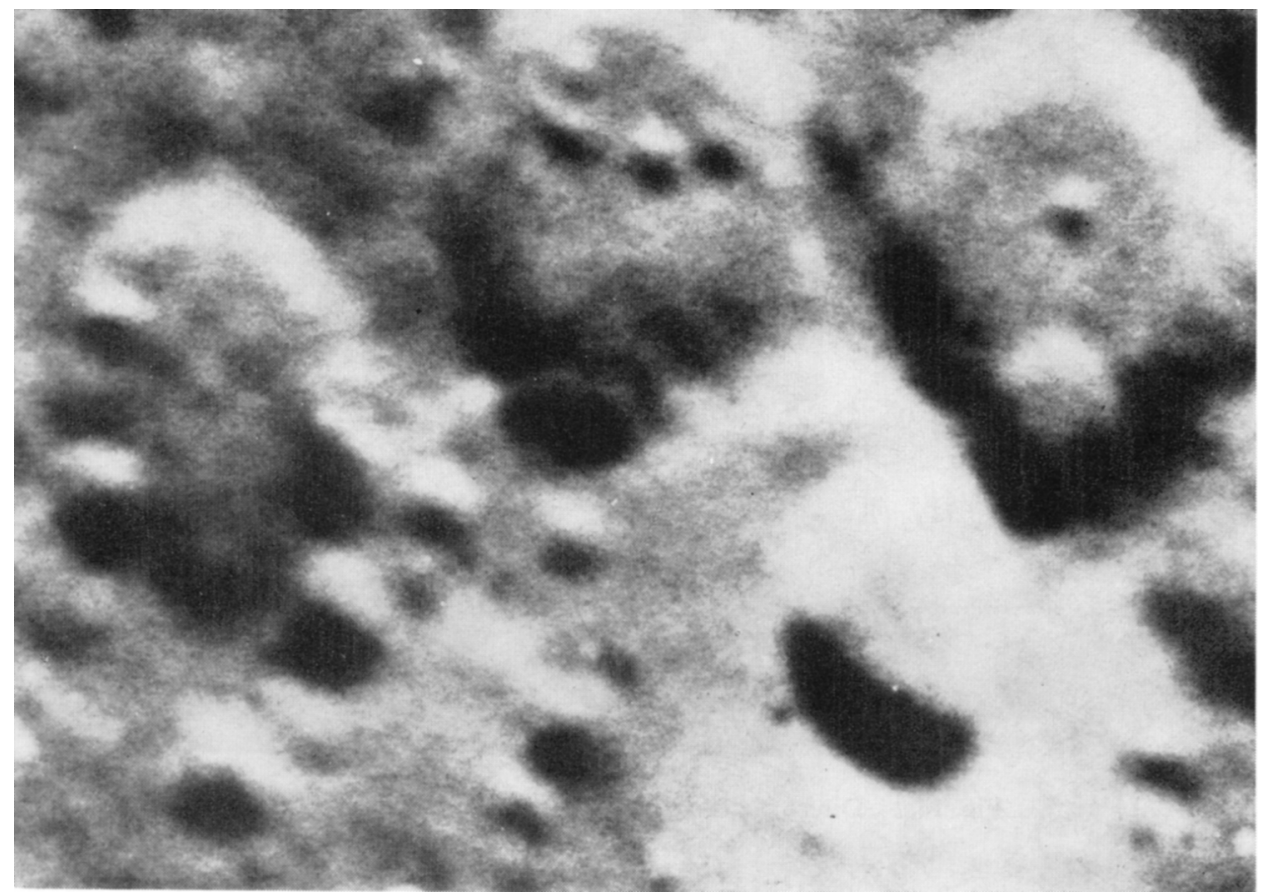

Fig. 14c. Reconstruction of (b), by Dr Vulmière. (Institut d'Optique - Paris).

We should not forget in the field of high spatial resolution the 26 in. Gregory $F / 502 \mathrm{E}=0.19^{\prime \prime}$.

2 cameras (1500-3000) and (4000-6500) of D. Allen, R. Becker and H. Zirin from the Caltech (Allen et al., 1970).

For these telescopes of small focal ratio, the choice of the array of lenses can give the possibility to use them for studying extended faint sources (high local ratio of the array of lenses, see Courtès (1967). As a matter of fact, it would be difficult to design for UV such an optical system similar to the focal reducer (Courtès, 1960; 1964). Array of lenses (some are magnesium fluoride and lithium fluoride or sapphire) is a very light and transparent solution for high focal ratio.

\section{Large Telescopes in Space}

Evaluation of the performance (limiting magnitude of stars) of such a telescope:

(a) the sensitivity is limited by the size of the diffraction ring correctly scanned by the spatial resolution of the tube (the number of lines to scan the Airy's function is not very well defined).

Extrapolations from Applied Optics and our own experiment with LAS 'night camera'

(Generation II)
A telescope diameter of $40 \mathrm{in.}$

40 in. $\quad 100 \mathrm{~cm}$

integration tube

1). $1000 \AA$ 


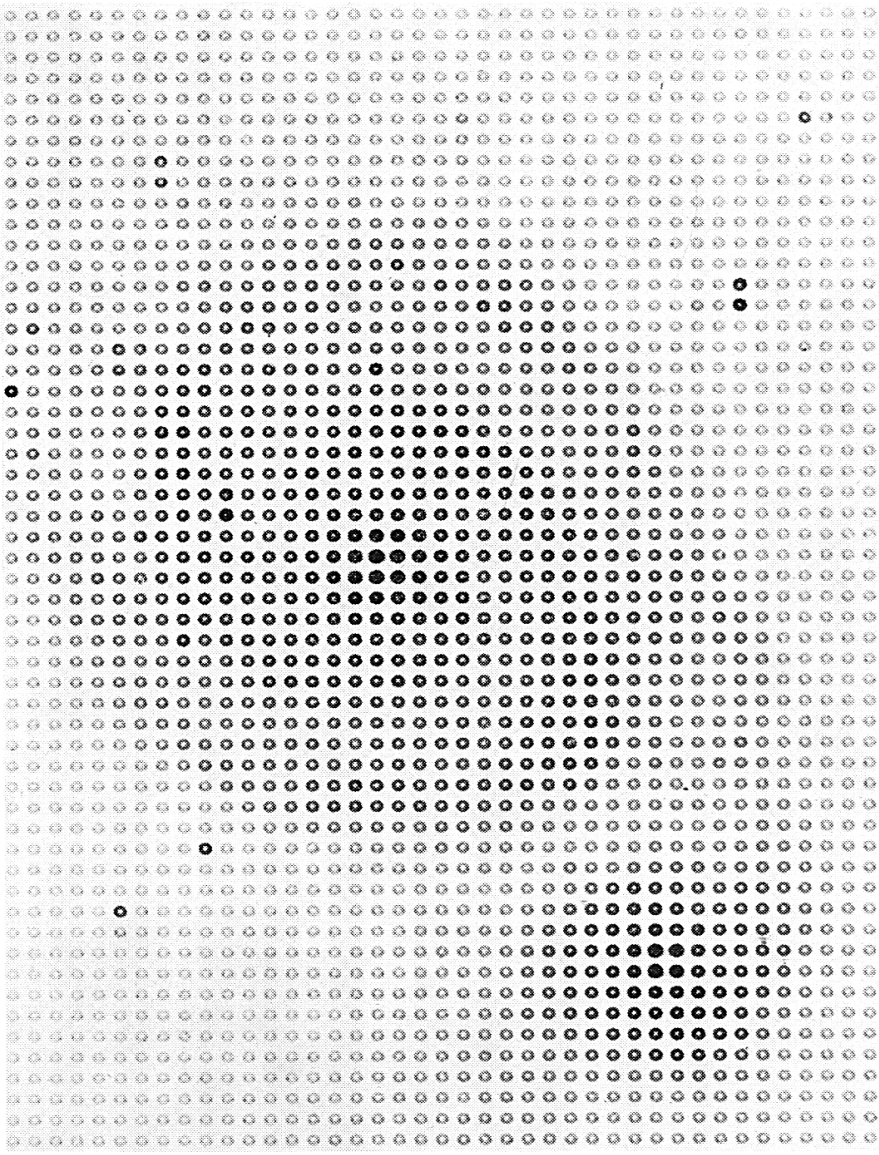

Fig. 15a. The galaxy M 51 - Array of lenses designed by M. Bonnet (Laboratoire du Film Gaufré du CNRS, Paris) - Central obscuration of the pupil due to the secondary mirror. Photograph by G. Monnet and Y. Georgelin.

Exposure time and magnitude reached

\section{Extended objects}

Nucleus of galaxies

Structures of nuclei of about $0.5^{\prime \prime}$ diameter

$$
\begin{aligned}
2 \mathrm{~s} \rightarrow m & =14 \\
5 \mathrm{~min} \rightarrow m & =19 \\
30 \mathrm{~min} \rightarrow m & =21
\end{aligned}
$$

$E=0.1$ " jets, filaments ( $M 82$,

M 87), double stars, planets, etc...

$60 \mathrm{~min} \rightarrow m \cdots 21$

$60 \min >m \quad 18$

Several nuclei of compact galaxies should be reached. 


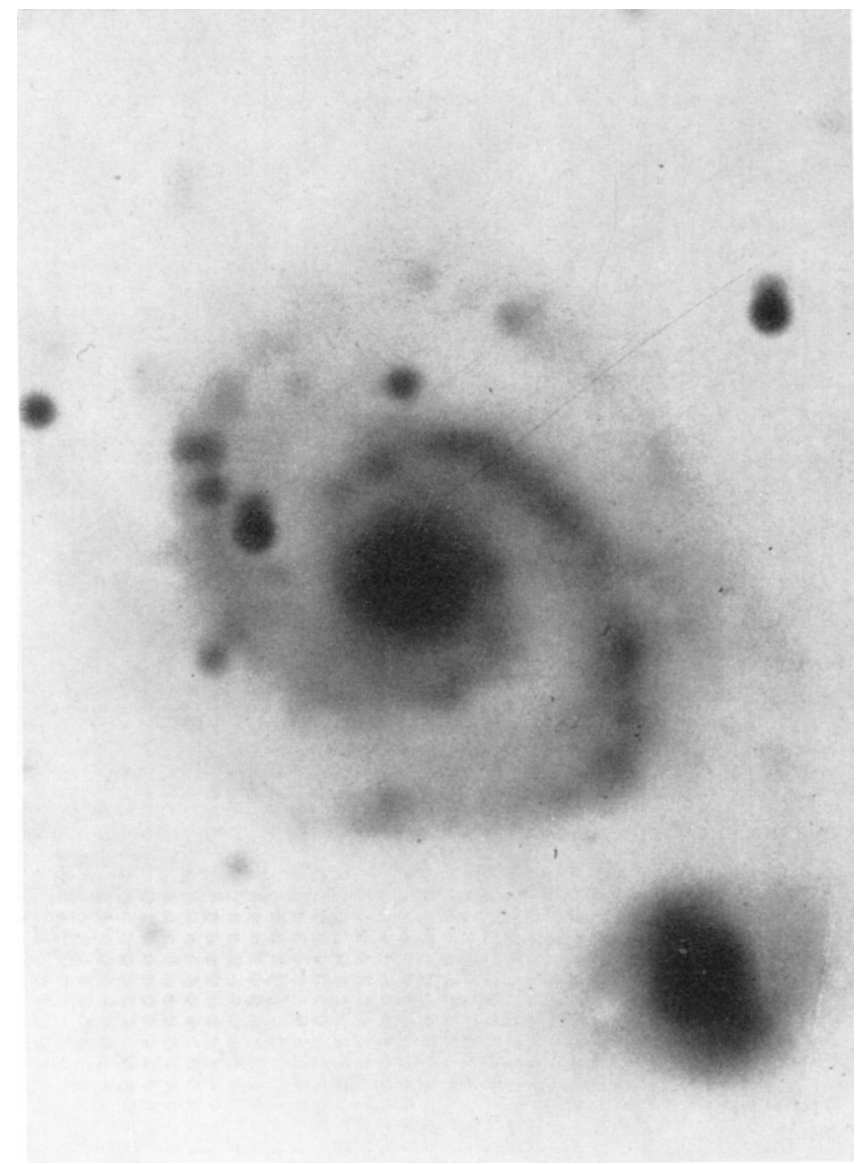

Fig. 15b. The galaxy M51 - Reconstruction from the plate a, obtained by Dr Vulmière (Institut d'Optique de Paris) - The resolution is of about 10". Some performances of this order could be obtained from Generation III space telescopes.

(b) the sensitivity is evaluated for the first ring of the same diameter as the resolution of the scanning.

$$
\begin{aligned}
& \text { The photometry would be poor but the } \\
& \text { sensitivity could reach } m=26 \text {. } \\
& \text { Interesting for survey of the 'Empty } \\
& \text { Regions', the Apollo Telescope Mount } \\
& 120 \text { in. could reach } m=28 \rightarrow 29
\end{aligned}
$$

Remark: If it proves impossible to reach such a performance, the M51 example given (Figure 15) shows the great interest of large telescopes in spite of their eventually insufficient guidance or optical quality $\left( \pm 6^{\prime \prime}\right)$.

\section{Selection of the Wavelengths}

Transmission: this selection can be made by transmission of some materials, for in- 
stance calcium fluoride or sapphire to cut $\mathrm{L} \alpha$ or classical colored filters for the near UV. The possibilities are very limited but very useful, combined with sensitivity curve of the photocathode.

\section{A. INTERFERENCE FILTERS}

Some begin to be convenient in the near UV but transmission and durability of their bandwidth should be improved. In any case, we must remind ourselves that the interference filters behave like a thin Fabry-Pérot interferometer. If one illuminates with a source of the same spectral function as the one to be used, one obtains (if the filter is correctly peaked) the first interference ring or the center spot. With the central obscuration of the secondary mirror (telescope) the case of the ring is the most frequent. The geometry of the optical system has to be designed in order to select, by the annulus-like entrance, the pupil or the first interference ring. In this condition, we reach the best compromise between transmissions and selectivity (Courtès, 1964; Courtès, 1962; Cruvellier, 1967).

\section{B. SELECTION BY REFLECTION COATINGS}

Very good coatings are now available. If the foot of the bandwith is still transmitting a few percents of the unwanted light, (Figure 16) it is always possible to use, for instance, the two reflections of the two mirrors combination telescope. LAS wide field PUV 'night camera' was coated in this way and the new Janus wide field LAS camera uses a system shown by Figure 17 (see similar design in Figure 20).

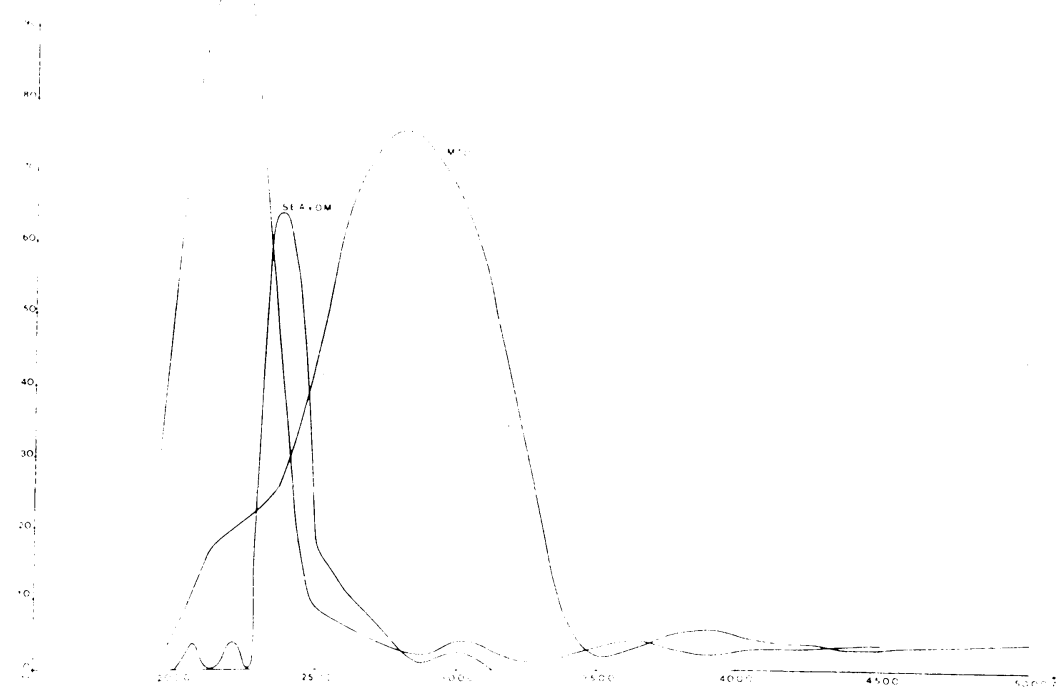

Fig. 16. Exampie of reflexion selective coatings in the near UV. OCLI (U.S.A.). SEAVOM and MTO (France). 


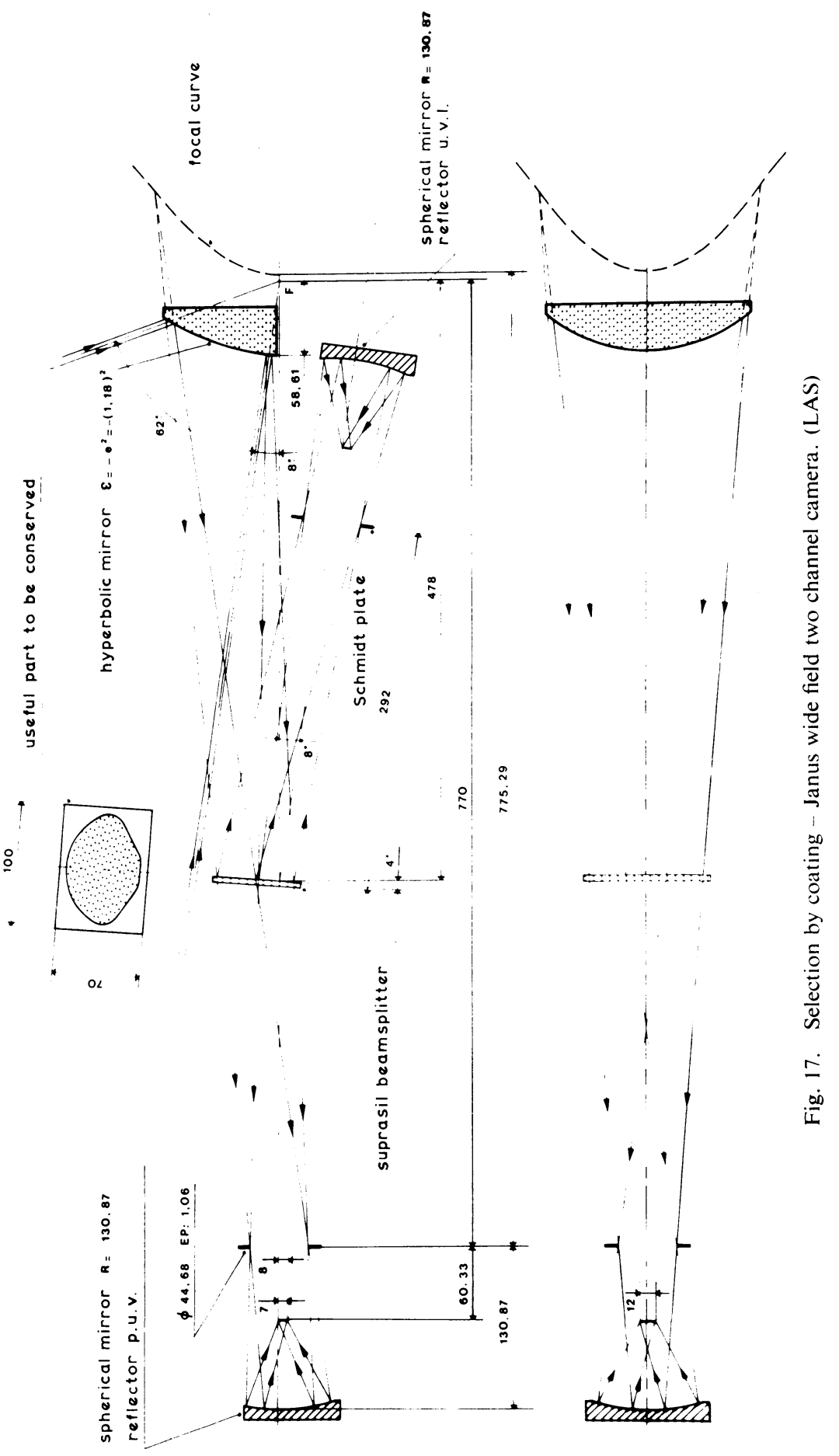


A new Swiss design, from Geneva Observatory* gives a good definition of the bandwidth, using four successive reflections (Figure 18). In spite of these reflections, the transmission of the 'filter' is of about 0.70 . The 'filter' is located in front of a small Schmidt camera. If the coating of $m_{2} \mathrm{~m}_{2}^{\prime}$ is chosen differently (another bandwidth) one can tilt one mirror of a very small angle. Double stars images appear in the field and colour indices can be obtained owing to direct photometric comparison on the same plate.

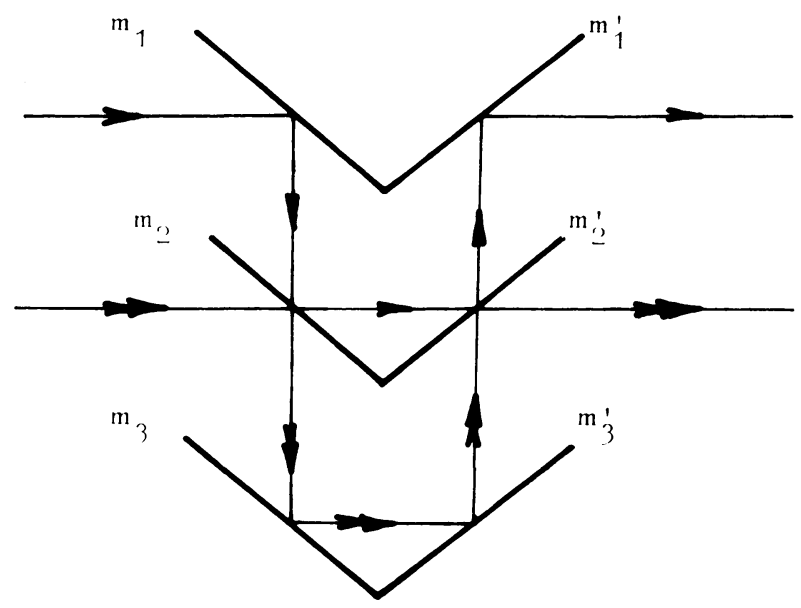

Fig. 18. Geneva Observatory - new reflection filter used for photographic photometry of stars in UV.

The same advantage is found for the 'Janus' experiment, a simultaneous recording of the possible drift of the images has a better photometric accuracy in the determination of the colour index, because of the same worm-shaped star images in the two bandwidths (2000 $\AA$ for $3500 \AA$ for Janus).

\section{Multibandwith Simultaneous Filter**.}

\section{SELECTION BY GRATING}

The main inconveniences of interference filters are their limited use in short wavelengths and the number of bandwidths limited practically to one. Gratings give a very convenient solution to this problem (Courtès, 1964; Courtès, 1962; Cruvellier, 1967). The principle is the following: one forms the image on a grating, the image of the pupil is dispersed in a spectrum, one selects any bandwith in this spectrum. In this filter the bandwidth is independent of the limit cycle fluctuation of the star image (Persee experiment application (Figure 10)). The illumination of them onochromatic exit pupil is uniform.

The spectral resolution is high when the focal ratio is small, the field depends on the

* Private communication from Prof. Golay.

** We call this optical design 'B.P.M. filter' (Bandes Passantes Multiples). 
grating diameter, holography grating offers new wide field solutions. This filter is mainly interesting for the instruments of the 4th generation, but gives an attractive system for guidance of the order of a few arc minutes. We use it with several cameras Courtès and Bonnet (1962) or we combine arrays of Fabry lenses with a BPM filter (Figures 19 and 20) (Atlas LAS S. 183 Apollo sky Lab. experiment).

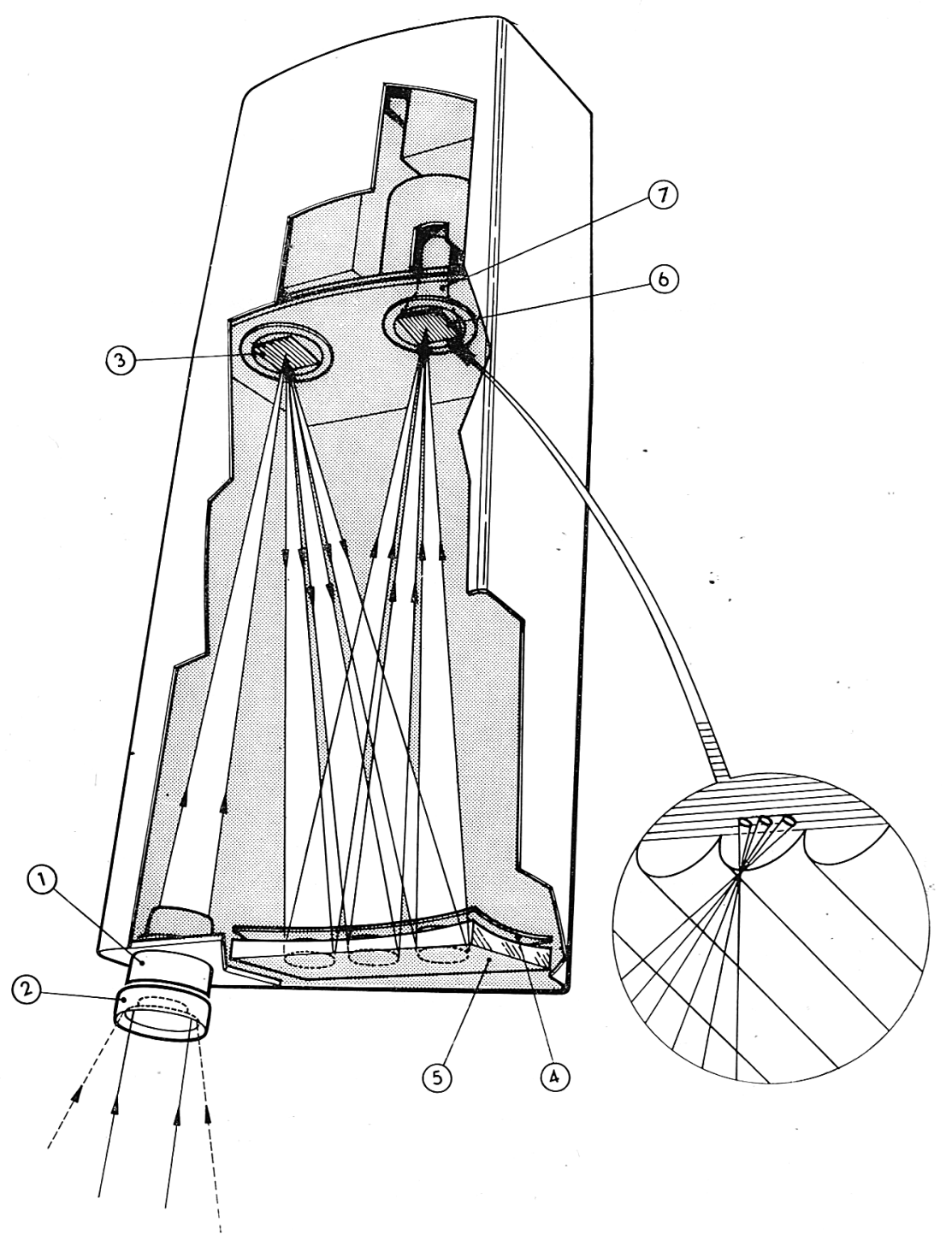

Fig. 19. Multi band width TV design, using a grating as a filter (BPM filter, CHROMOTRON) 1. objective -2 . additional lens -3 . grating -4 . selection diaphragms -5 . spherical collector $\cdots 6$. array of lenses - 7. TV integrating tube (the monochromatic pupils are scanned by the same line of the TV). 


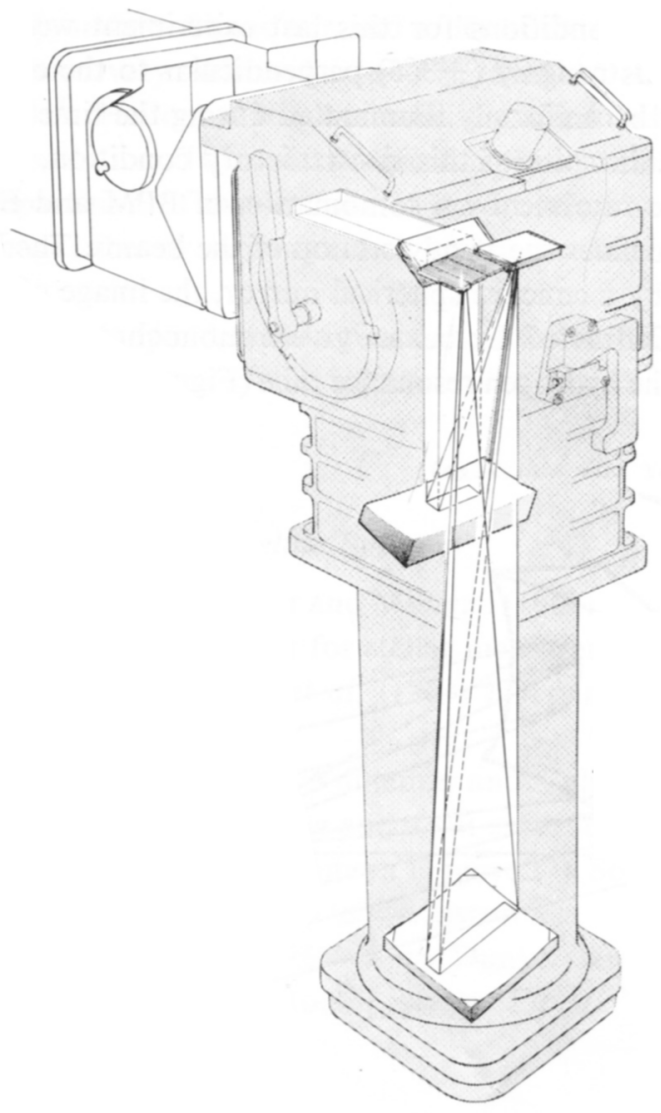

Fig. 20. The LAS S 183 Apollo Skylab experiment, mounted on the airlock of the skylab. The beams are folded by a flat 45 mirror in order to make easier the use by the astronauts (human constraints).

2. Other Space Experiments Founded on the BPM Filter

Several optical systems of this kind have been designed for the LAS (Marseilles) and the Service d'Aéronomie (Paris) by our group.

2 bandwidths Apollo S 183 Skylab-LAS experiment - 1800 to $3800 \AA$ (will fly end of of 1972) (Figure 20)

4 bandwidths Persée and Céphée LAS rocket experiments on M 31 galaxy and Orion Nebula - flown in 1968 and 1969 (Figure 10)

- Atlas LAS rocket experiment (will fly in 1971)

3 bandwidths - Sun images in the UV by Bonnet (limb darkening) (Courtès and Bonnet, 1962) rocket experiment (Aéronomie - Paris)

8 bandwidths Sun eclipse spectrophotometry by Blamont and Malique (Aéronomie Paris)

A peculiar case with 1 bandwitdh is the Sun $\mathrm{L} x$ iesonance tube monochromator by Blamont and Vidal Madjar(OSO 5 satellite) Aéronomie Paris. 
A priori observing conditions for this last experiment were very difficult. OSO rotates around its axis roughly $\left( \pm 3^{\circ} 5\right)$ perpendicular to the ecliptic. The resonance hydrogen tube must be uniformly illuminated during the time of crossing of the sun into the instrument. In spite of this contradictory condition, we proposed that the difficulties should be resolved by a combination of BPM and Ebert Fastie systems. The entrance pupil defines the steady section of the beams. The beams oscillating by $\pm 3^{\circ} 5$ are collected by a concave spherical mirror, the image of the Sun is formed on the plane grating. Diffracted $\mathrm{L} \alpha$ beams give a monochromatic steady image of the entrance pupil into the hydrogen resonance tube (Figure 21).

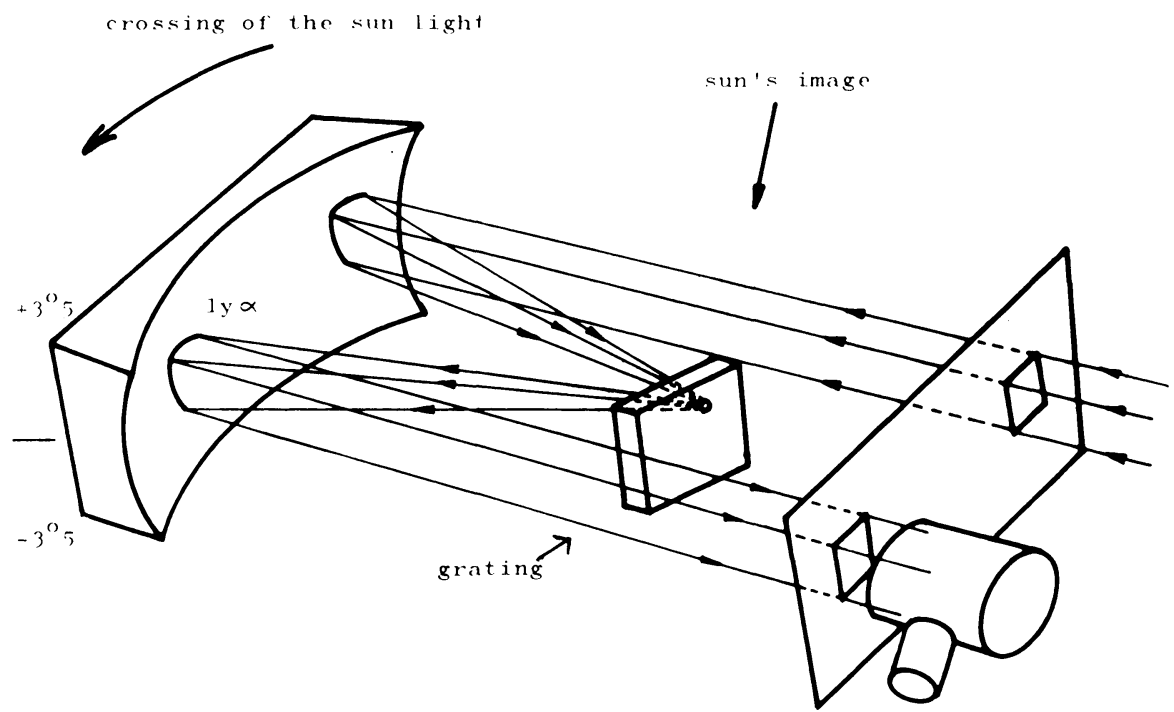

hydrogehe tube

Fig. 21. OSO $5 \mathrm{La}$ Experiment. (Steady La pupil in spite of $\quad 35$ acquisition) from the Service d'Aeronomie du CNRS. Paris.

\section{Spectrographic Technics}

The first dream of astronomers has been to obtain spectra of the celestial bodies from outside the atmosphere. First, the sun has been studied owing to the beautiful observations (spectra and spectroheliograms) of NRL by Tousey. (Tousey will describe his very ingenious instrumentation during this Symposium). If we follow our classification founded on stabilization, we see that the spectral resolution will be dependent of the guidance, in most cases high resolution will need fine guidance. However, one often forgets some very useful optimisations. Final focal length of the system has to be as short as possible (sharp images* in spite of poor guidance), then

* Remark: (a) sharp spectra in case of objective grating; and (b) total flux admitted in case of slit spectrometer. Consequently the final focal ratio will be very high but we saw several good catadioptric high focal ratio cameras. 
the angular dispersion (number of lines/mm and order of the grating) must be as high as possible. This has been very well understood in the OAO Goddard $12001 / \mathrm{mm}$ between 3000 and $8000 \AA$ (Hallock, 1962) but echelle grating and crossed dispersion, give a better spectral resolution. This solution has long been familiar to astronomers. The square-shaped repartition of the sections of the spectrum is particularly convenient for the use of image tubes or memory tubes, giving another reason to choose this solution.

We classify some spectroscopic design in function of the stabilization - such as we did for sky image instrumentation.

\section{Generation I}

(a) Scanning by Balloon, Rocket or Satellite Rotation

1. stellar spectrophotometer of Stecher and Milligan (1962).

A rocket-borne objective spectrometer for stellar measurements between 1300 and $4000 \AA$ has been designed by Blakney et al. (1961) $F / 1$ parabolic and scanning by rotation of the rocket.

2. Night sky balloon spectrometer by Vuillemin and Cohen (LAS). It is a "night sky' PUV camera, equipped with a grating and a slit extended from 10 to $90^{\circ}$ zenith distance owing to the wide field of the camera (Figure 22). Some good spectra of $\mathrm{O}_{2}$ Hertzberg bands have been obtained owing to this instrument.

3. Zenith Night glow experiment at $2600 \AA$ by Dunkelman 1969. A value of the night glow has been measured and found about $2 \times 10^{-5} \mathrm{erg} \mathrm{s}^{-1} \mathrm{sr}^{-1} 100 \mathrm{~A}^{-1}$ (2600 $\AA$ ).

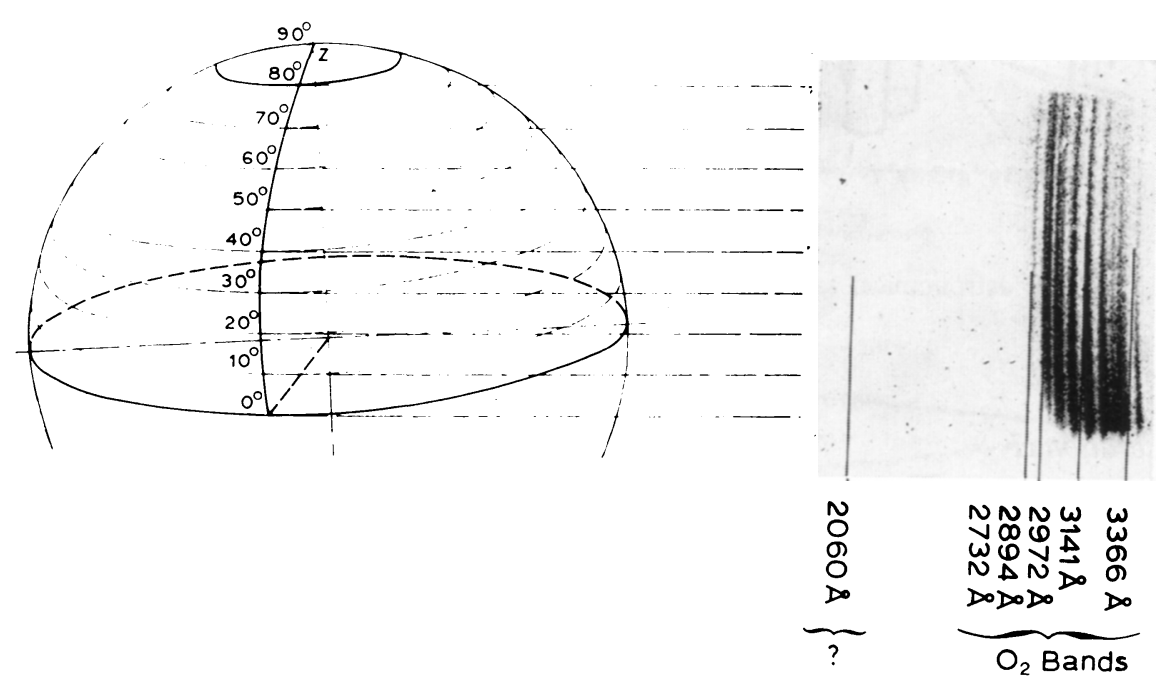

Fig. 22. Wide field slit spectrum of the night sky from a balloon flying at an altitude of $38 \mathrm{~km}$. One see the Zenith Horizon effect in the brightness of the bands. 


\section{(b) Satellites}

Satellite experiments D2A (Laboratoire d'Aéronomie TD1 (Liège - Edinburgh) will be described in this Symposium by their authors.

D2B - We give a short description of the LAS D2B experiment. D2B is an astronomical satellite, its small size forces the choice of a very simple optical design (Figure 23) in order to improve its efficiency. Two concave gratings, one for each wavelength range ( 800 to $2800 \AA$ ) and (1500 to $3500 \AA$ ) give spectra of the stars scanned by two photomultipliers for each range of wavelengh. This very simple system designed by $P$. Cruvellier is equivalent to a $30 \mathrm{~cm}$ conventional telescope followed by a full range of wavelength spectrometer. In addition to these stellar performances, D2B will measure four bandwidths of the sky background (galactic repartition and interplanetary dust repartition). Baffle has been previously described (Figures 3 and 4).

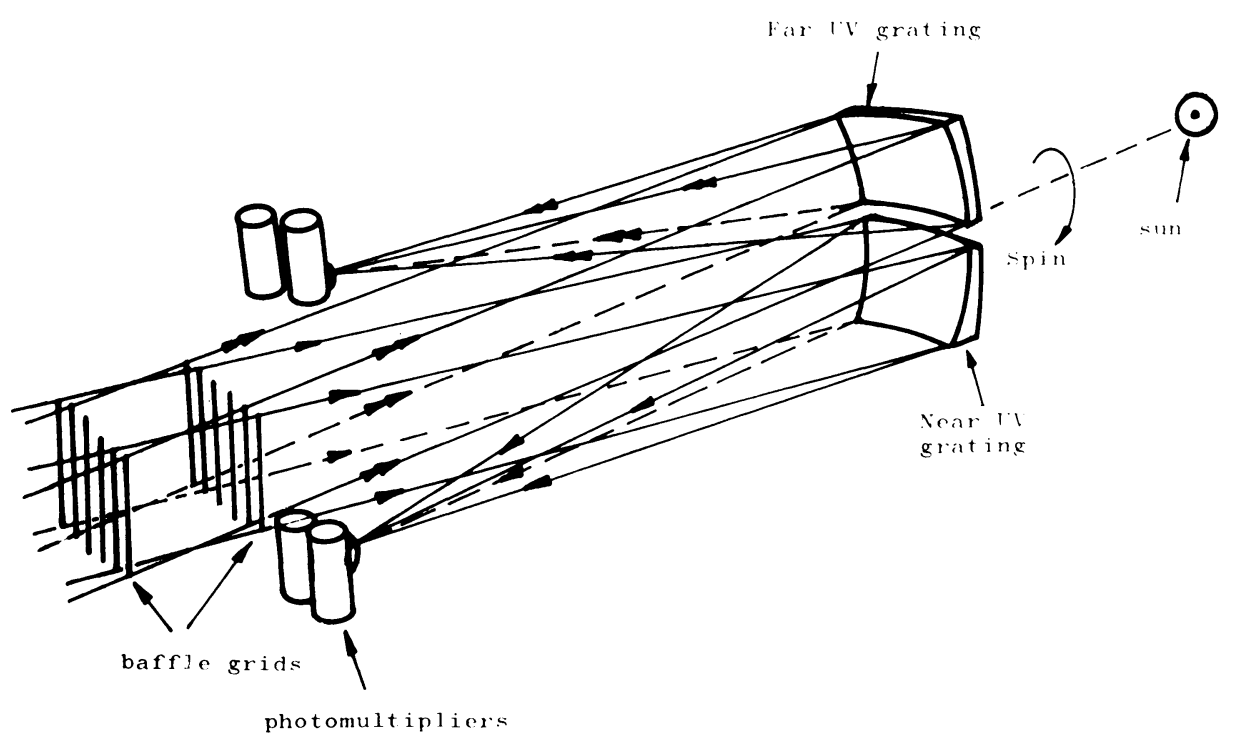

Fig. 23. $\mathrm{D}_{2} \mathrm{~B}$ astronomical satellite. Four simultaneous bandwidth obtained owing two concave gratings and a common baffle.

\section{Generation II}

\section{(a) Spectrometer of Gemini 11}

$U V$ spectrophotometry of Canopus owing to an objective prism from Gemini 11 by Kondo et al. (1970). This instrument is a short focal length camera of $75 \mathrm{~mm}$ and $22 \mathrm{~mm}$ aperture, equipped with a grating of $600 \mathrm{l} / \mathrm{mm}$, dispersion is $183 \AA / \mathrm{mm}$, with a field of $30^{\circ}$. Stabilization is in the hands of the astronaut.

(b) The Michelson-Connes interferometer proposed by Dr Clark is a good com- 
promise to obtain a high resolution, in spite of a poor stabilization, owing to its large 'slit function'. It is fully described by Dr Clark in this Symposium. The accuracy of parallelism of the plates is of the same order as the needed guidance, but the acquisition field is larger. This instrument can be used on various spacecrafts and should be well adapted to the conditions of 'Generation II' guidance and large spacecrafts (Skylab, space platforms, etc....).

(c) Fabry-Pérot design such as the spherical interferometer of P. Connes has been devoted to the red OI night glow lines by Blamont and Luton, but could be used in some near UV experiments with convenient reflection coatings. The wide 'slit' function of the Connes' Fabry-Pérot is the way to obtain high resolution with Generation II and III vehicles.

\section{Generation III}

(a) Rocket measurements of UV spectra of Venus and Jupiter (1200 to $1800 \AA)$ (Moss et al., 1969). This observation is made with a telescope of $36 \mathrm{~cm}$ diameter, on Aerobie rocket, a monitorized secondary mirror secures a guidance of $\pm I^{\prime \prime}$. Two original solutions: lithium fluoride prism as dispersive element and a wheel selecting several bandwidths in the spectrum.

(b) Small LiF prism spectrometer $\left(15^{\circ}\right)$ for space application proposed by $\mathrm{H}$. W. Moos, R. C. Vitz, J. R. Barry and J. L. Backley. Aerobie (1969) (Lemaire, 1970). The authors show that a conventional Ebert-Fastie spectrometer has a transmission of $\mathrm{O}, 15$ in $\mathrm{L} x$. They prefer the original optical design of Figure 24.

(c) We find some very important astronomical results, the discovery of the expansion shift of absorption lines in 6 stellar spectra in Orion with a resolution of $3 \AA$. by

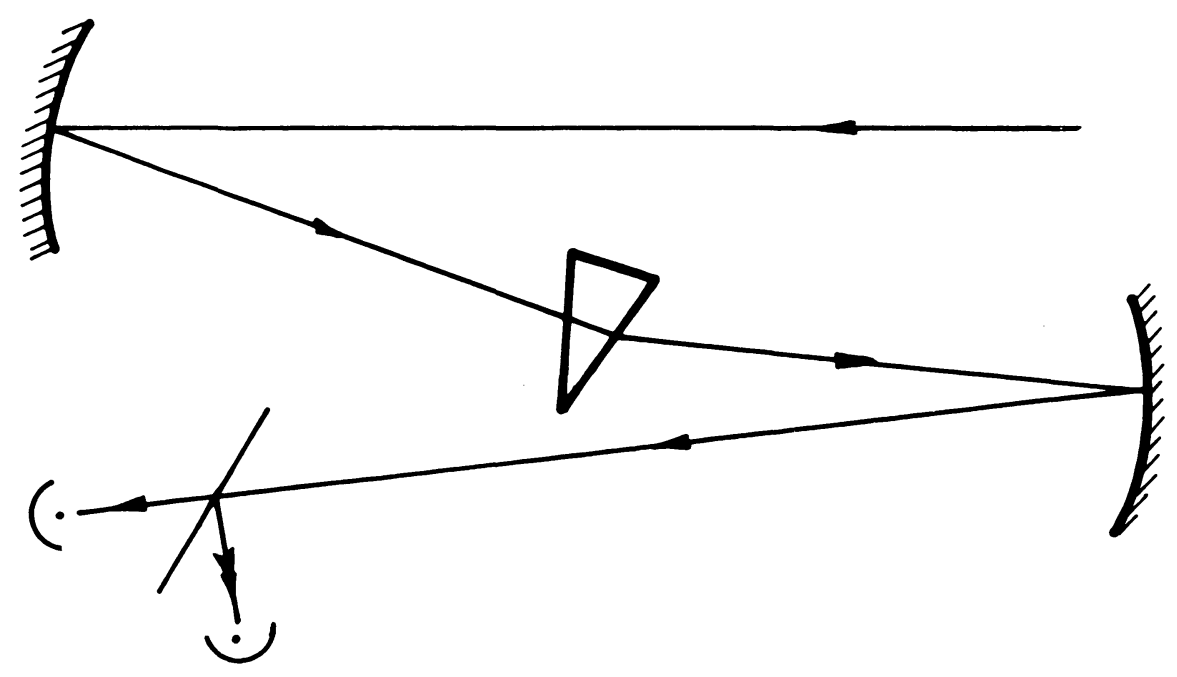

Fig. 24. Small prism spectrometer for space application proposed by Moss et al. (1968). 
Morton (1967). The optical design consists in a Schmidt 'Generation II' camera equipped with objective grating. The experiment is monotorized on one direction, perpendicular to the dispersion.

(d) An original objective prism in front of a Ritchey Chretien $F / 2.5$ telescope, designed by K. Henize, Jim Wray, O. Callaghan for the A pollo Skylab. This telescope is pointed on the sky owing to an external mirror designed by $\mathrm{O}$. Callaghan to be used by the Henize experiment and S 183 Sky panorama LAS experiment on one of the airlocks of the Skylab (Figure 20).

Both experiments use photographic emulsions.

(e) The multichannel spectrometer of Stechert consists of a Cassegrain telescope followed by a grating monochromator. This experiment has given a great deal of spectral functions of stars at 5 and $10 \AA$ resolution.

(f) Electronic camera on Schwartzschild $7^{\circ} 5$ field telescope equipped with four $12001 / \mathrm{mm}$ gratings used by Carruthers (Fig. in Carruthers (1968). 16 stars have been observed up to the 4.6 magnitude between 950 and $1400 \AA$. Exposures were 3 to $10 \mathrm{~s}$.

(g) Experiment with concave $12001 / \mathrm{mm}$ grating and $14 \mathrm{~cm}^{2}$ aperture has given to Smith (1969) the spectroscopy of $\varrho$ Puppis below $1100 \AA$ with a resolution of $0.8 \AA$.

\section{Generation IV}

\section{Recent UV Solar Spectra and Spectroheliograms}

(a) Excellent photographs of the sun in He II $304 \AA$ line and MgIX $368 \AA$ have been obtained by W. E. Austin, J. D. Purcell, C. B. Snider, R. Tousey and K. C. Widing, with a normal incidence grating giving a dispersion of $8.3 \AA / \mathrm{mm}$. The resolution was of about $\pm 10^{\prime \prime}$.

(b) Some performances, but in the near UV, have been given by the BPM filter of Bonnet and Courtès described before (this volume, p. 291).

(c) Echelle grating and crossed dispersion is one of the best solutions for this kind of instrument*. Bonnet, Lemaitre and Samain from Paris (Lemaire, 1970) obtained high resolution spectra of MgII $2800 \AA$ and C $1994 \AA$ line on the Sun. Dr Reay from Culham (this volume, p. 254 and 304) designed a high resolution spectrograph founded on the same concept.

(d) Extreme UV spectroheliometer (170 to $400 \AA$ ) designed by J. H. Underwood, J. D. Mangus and W. M. N. Neupert (NASA) using a spherical grating on glancing incidence $\left(77^{\circ}\right)$ with an aperture of $11 \mathrm{~cm}^{2}$ and a resolution of $10^{\prime \prime}$.

(e) Glancing incidence spectrometer for far UV between 200 and $1200 \AA$ is also designed by Delaboudinière for the French satellite D2B**.

(f) Original solution in high resolution is given by a Belfast Culham (Bates et al., 1970) spectrograph using Fabry-Pérot interferometer on $2800 \AA$ lines.

* Echelle grating provides a very high angular dispersion. Then the focal length of the camera can be as short as possible and the conditions of guidance can be less severe for a given linear dispersion (see p. 294).

** Private communication. 


\section{Combination of Selectivity and Spectrographic Technics}

Non-classical use of gratings in converging beams gives interesting solutions in some special cases:

(1) OGO-5-L $\alpha$ experiment of Bertaux and Blamont (1971) (Service d'Aéronomie du CNRS) consists in a step by step plane mirror $M_{1}$ scanning the $L \propto$ geocorona from the very eccentric orbit of this satellite. Parallel beams, collected by a parabolic mirror $\mathrm{M}_{2}$ give an image of a small field $\left(40^{\prime}\right)$ of the geocorona selected by a hole made in the flat $M_{1}$ mirror (Figure 25).

The natural astigmatism of the plane grating $G$, used in converging beams, is corrected by an aspherical concave off-axis mirror $\mathbf{M}_{3}$.

A bandwidth of $80 \AA$ around $\mathrm{L} x$ line is selected by a diaphragm in front of the hydrogen tube.

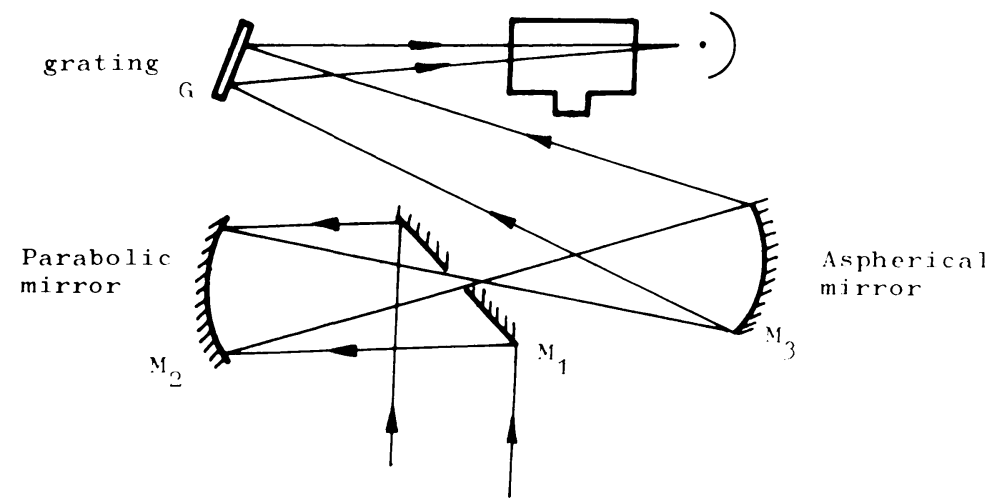

Fig. 25. OGO $5 \mathrm{~L}$, experiment on the Geocorona by Bertaux and Blamont.

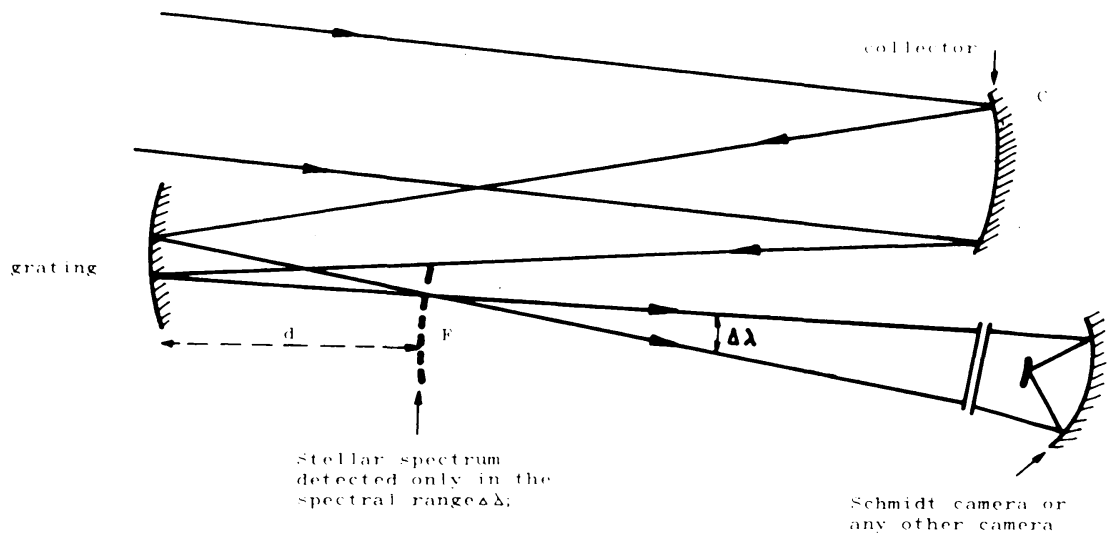

Fig. 26. High contrast spectrographie BPM filter. This instrument gives an image of the sky, similar to the conventional objective-grating but only in the predetermined range $3 \lambda$. Several hand width can be obtained simultaneously. 
This experiment has given the beautiful observation of the wide $\left(9^{\circ}\right) \mathrm{L} \alpha$ glow of the Comet Bennet - 1970 .

(2) Limited spectral range spectrography (spectrographic BPM filter). A new optical combination derived from the grating filtering (BPM filter) can give spectra of some ranges of wavelengths, eliminating consequently the not wanted wavelengths (visible light, for instance, or some too bright parts of the spectrum when one wants to detect faint spectrographic features) (Figure 26).

The range of wavelengths $\Delta \lambda$ is selected as in the BPM filter, but the focal surface of the collector or telescope $\mathrm{C}$ is no longer on the grating but at a distance $d$. The star images in $\mathrm{F}$ are short spectra, limited to the range $\Delta \lambda$. The sky background is of course not dispersed but contributes to the fog only for the range $\Delta \lambda$, the limit magnitude increases. The probability of superposition with the spectra of the other stars of the field is greatly diminished.

\section{Conclusion}

We have described a part of the great effort to obtain images and spectra of the celestial bodies. The difficulties of the subject have been deeply stimulating for UV optics and new ingenious solutions have been found.

Now what will be the optics of the very large space telescopes of space stations? Maybe those optics will come back to the sometimes heavy solutions of groundbased Astronomy, but the present effort has not been useless.

We are convinced that the problem of a telescope such as $120 \mathrm{in}$. Apollo is one of the most exciting of these coming years, because it could be the absolute weapon of Astronomy; with a resolving power ten times higher than the $200 \mathrm{in}$. telescope of Mount Palomar (under the best atmospheric conditions). Stars a hundred times fainter would be detected. The study of individual stars should not be restricted to the nearest galaxies of the local group.

Very distant objects should be detected to test the different cosmological models of the Universe.

Before this very powerful telescope, some simple optical designs, as it is shown in this paper, could give, even with modest rockets and photographic telescopes, better performances than the present astronomical satellites. Improvements owing to the image tubes or the Lallemand's electronic camera used by Carruthers will reach the top of the sensitivity of this rocket program.

Unfortunately, recovery is needed in both cases and that is not an optical problem.

\section{References}

Baranne, A., Detaille, M., and Lemaitre, G.: 1969, in J. Home Dickson (ed.), Optical Instruments and Techniques, Oriel Press, p. 311.

Baranne, A.: 1966, in D. L. Crawford (ed.), 'The Construction of Large Telescope', IAU Symp. 27, 311.

Bates, B., Bradley, D. J., McKeith, C. D., Keith, N. E., Burton, W. M., Paxton, H. J. B., Shenton, D. B., and Wilson, R.: 1970, in L. Houziaux and H. E. Butler (eds.), 'Ultraviolet Stellar Spectra and Related Ground-Based Observations”. IAU $S_{1}$ mp. 36, 274. 
Bertaux, J. L. and Blamont, J. E.: 1971, Astron. Astrophys. 11, 200.

Billingsly, F. C.: 1970, Appl. Opt.9, 289.

Blakney, R. M., Murty, M.V.R.K., Hochgraf, N., and Standenmaier, W.: 1961, Proceedings of the Conference on Optical Instruments and Techniques, Chapman and Hall, London.

Bowen, I.: 1967, Ann. Rev. Astron. Astrophys. 5, 45.

Burch, C. R.: 1942, Monthly Notices Roy Astron. Soc. 102, 159.

Carruthers, C. R.: 1968, Astrophys. J. 151, 269.

Chretien, H.: 1922, Rev. Opt. 1, 49.

Couder, A.: 1926, Compt. Rend. Acad. Sci. Paris, 183, II, 1276, p. 45.

Courtès, G.: 1960, Ann. Astrophys. 23, 115 to 217.

Courtès, G.: 1962, Compt. Rend. Acad. Sci. Paris 254, 1738.

Courtès, G.: 1964, Astron. J. 69, 325.

Courtès, G.: 1967, Compt. Rend. Acad. Sci. Paris 262, 1757, serie B.

Courtès, G. and Bonnet, R. M.: 1962, Ann. Astrophys. 25, 36.

Cruvellier, P.: 1967, Ann. Astrophys. 30, 1059.

Cruvellier, P., Roussin, A., and Valerio, Y.: 1970, in L. Houziaux and H. E. Butler (eds.), 'Ultraviolet Stellar Spectra and Related Ground-Based Observations', IAU Symp. 36, 130.

Davis, R. J. and Rustzi, O. M.: 1962, Appl. Opt.1, 131.

Dunkelman, L.: 1969, JOSA 59, 514.

Hallock, H. B.: 1962, Appl. Opt. 1, 155.

Jenkins, E. and Morton, D.: 1967, Nature 215, 1257.

Kondy, Y., Henize, K. G., and Kotila, C. L.: 1970, Astrophys. J. 159, 927; and in L. Houziaux and H. E. Butler (eds.), 'Ultraviolet Stellar Spectra and Related Ground-Based Observations', IAU Symp. 36, 180.

Kupperian, J. E., Boggess, A., and Milligan, T.E.: 1958, Astrophys. J. 128, 453.

Lemaire, P.: 1970, in L. Houziaux and H. E. Butler (eds.). UItraviolet Stellar Spectra and Related Ground-Based Observations', IAU Symp. 36, 250.

McGrath, J. F. and Marwitt, M.: 1969, Appl. Opt. 8, 837.

Monnet, G.: 1970, International Summer School of Space Optics (C.I.O.) Courtès, G. and Maréchal, A.

Morton, D. C.: 1967, Astrophys. J. 147, 1017.

Moss, Warren, Fastie, William, G., and Bottema, M.: 1969, Astrophys. J. 155, 887; JOSA (1968), p. 732.

Paul, F. W. and following papers: 1970, Appl. Opt.9. 243.

Rogerson, J.: 1963, Space Sci. Rev. 2, 261.

Runsey, N. J.: 1969, in J. Home Dickson (ed.), Optical Instruments and Technicules, Oriel Press, p. 5141.

Schwarzchild, K.: 1905, Astron. Mitt. Part 2.

Smith, A. M.: 1969, Astrophys. J. 156, 93.

Sivan, J.-P. and Viton, M.: 1970, in L. Houziaux and H. E. Butler (eds.), Ultraviolet Stellar Spectra and Related Ground-Based Observations', IAU Symp. 36, 120

Wynne, C.: 1969, J. Opt. Soc. Am. 59, 572.

\section{DISCUSSION}

R. Muri: What kind of image tube did you use for your measurements?

$G$. Courtès: Up to now we used only conventional photographic emulsion. 